\title{
GOING VIRAL: UNMASKING THE SPREAD OF NEW VOICES THROUGH ANALYZING
}

COVID-19 MEMES

Laavanya Srichandramohan

BA, University of Toronto, 2018

A Major Research Paper

presented to Ryerson University

in partial fulfillment of the requirements for the degree of

Master of Professional Communication

Toronto, Ontario, Canada, 2020

(C) Laavanya Srichandramohan, 2020 


\section{AUTHOR'S DECLARATION}

I hereby declare that I am the sole author of this Major Research Paper. This is a true copy of the MRP, including any required final revisions, as accepted by my examiners.

I authorize Ryerson University to lend this MRP to other institutions or individuals for the purpose of scholarly research.

I further authorize Ryerson University to reproduce this MRP by photocopying or by other means, in total or in part, at the request of other institutions or individuals for the purpose of scholarly research.

I understand that my MRP may be made electronically available to the public. 


\begin{abstract}
Internet memes are becoming a progressively more popular method of quick and easy communication. In this MRP project I will examine memes as a distinct method of digital communication. More specifically, my research paper will analyze the use of memes during the COVID-19 crisis of 2020, and whether memes can provide a comforting and relatable medium for dealing with public anxieties and for communicating complicated issues to large audiences. An analysis of which voices are amplified using the meme medium will also be crucial in understanding its communicative capability. I will also be analyzing which audiences most resonate with this new form of communication and how data on the popularity of memes can provide us with a better understanding of their limits and potential. Further research within this field of study is extremely relevant, and can aid in analyzing and evolving communicative practices in the foreseeable future
\end{abstract}




\section{ACKNOWLEDGEMENT}

Thank you to my supervisor Dr. Matthew Tiessen for your continuous feedback and support throughout the entirety of my MRP. I would also like my second reader, Dr. Robert Clapperton for providing your feedback and expertise to refine my paper. Finally, thank you to Ryerson University and the Department of Professional Communication in the Faculty of Arts and Design for providing me with the necessary resources to make my research process as easy as possible. 


\section{TABLE OF CONTENTS}

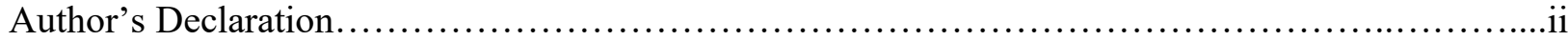

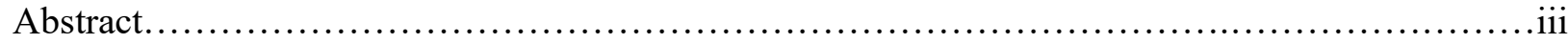

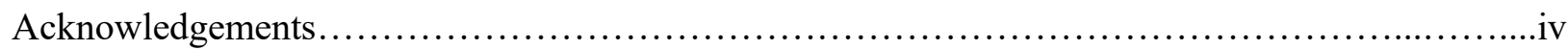

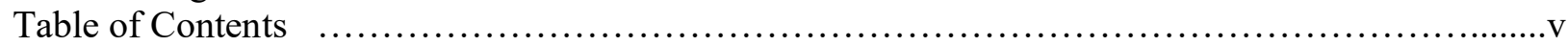

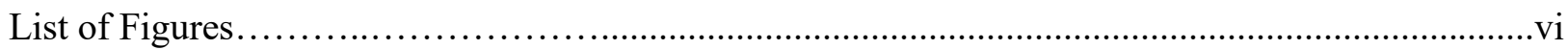

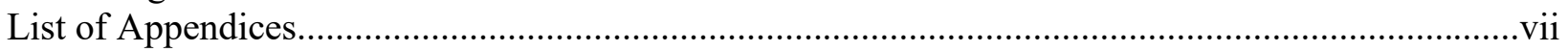

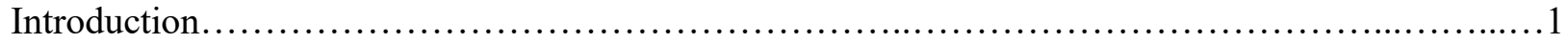

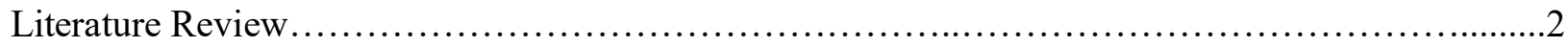

Media Consumption in Relation to Memes................................................

Memes as a Coping Mechanism .........................................................

Memes vs. Mainstream Media....................................................................................

Memes and the Emergence of Unheard Voices.....................................................................

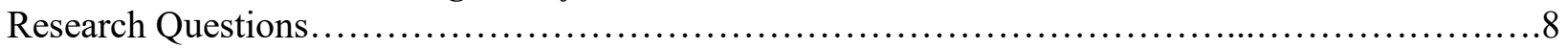

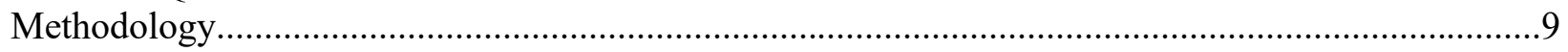

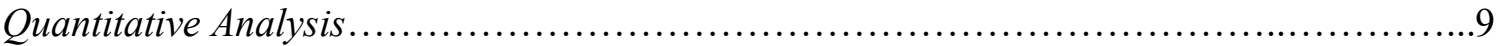

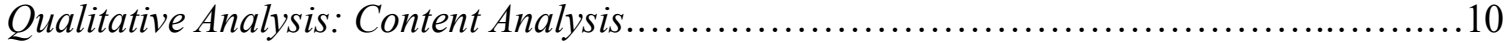

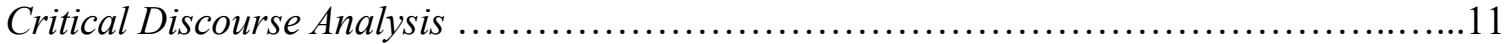

Selected Social Media Platforms for Analysis ..............................................12

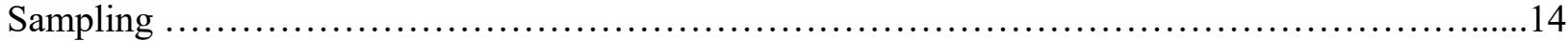

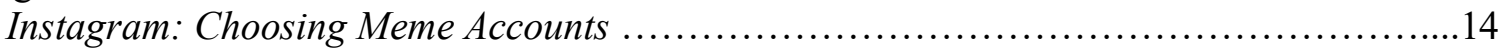

Instagram: Choosing Media Stories ..................................................16

Instagram: Quantitative Analysis....................................................................................17

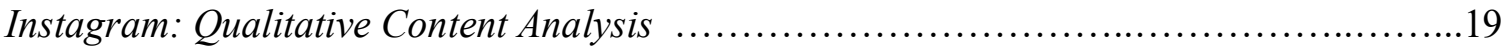

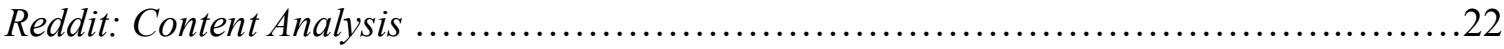

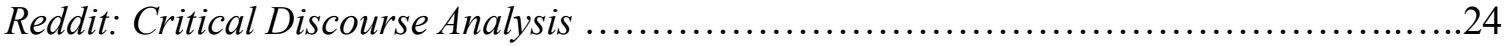

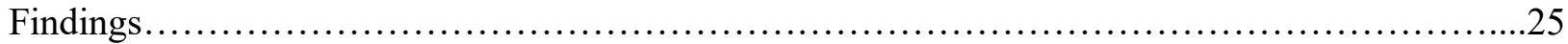

Instagram: Quantitative Analysis - Broad Observations ................................25

Instagram: Quantitative Analysis - Relationship with Followers.............................29

Instagram: Quantitative Analysis - Understanding Likes and Comments ......................30

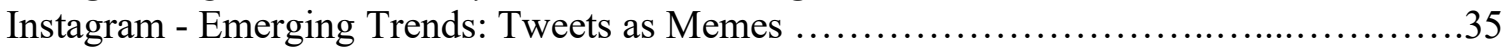

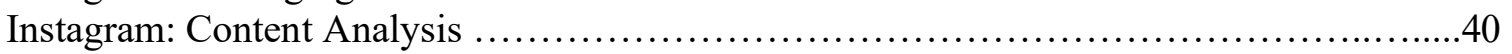

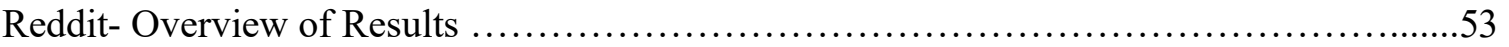

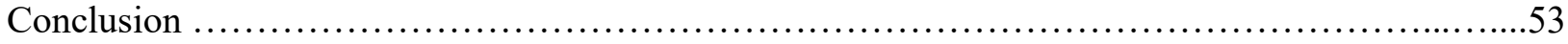

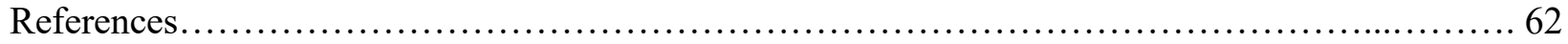




\section{LIST OF FIGURES}

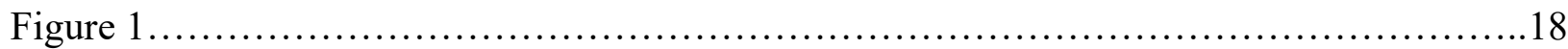

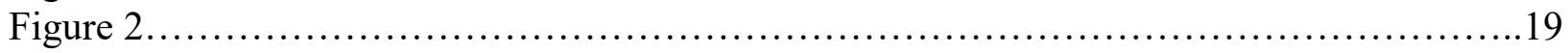

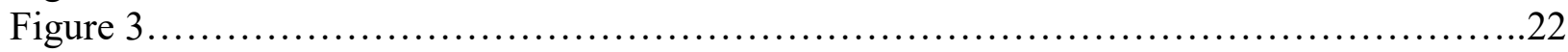

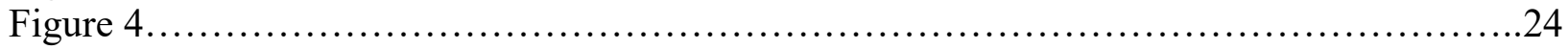

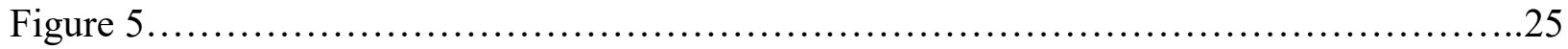

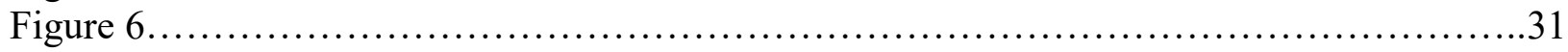

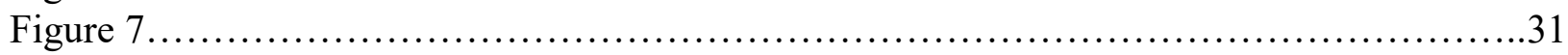

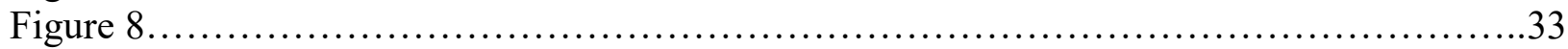

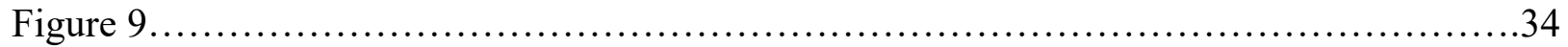

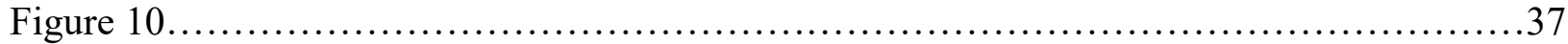

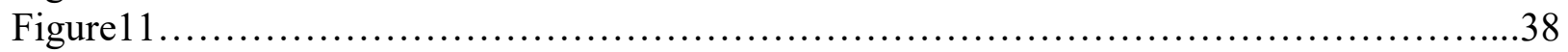

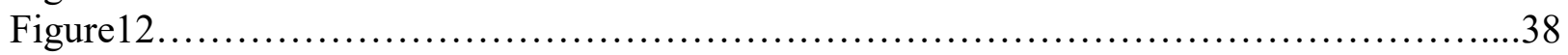

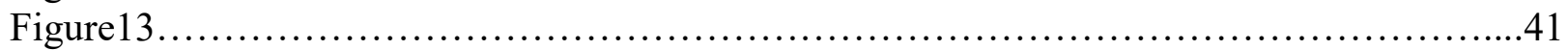

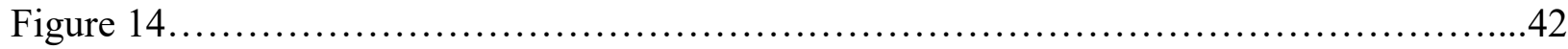

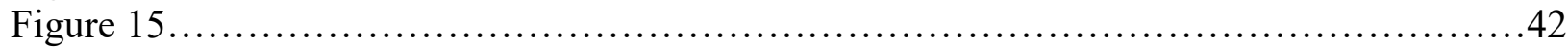

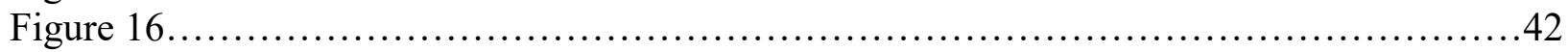

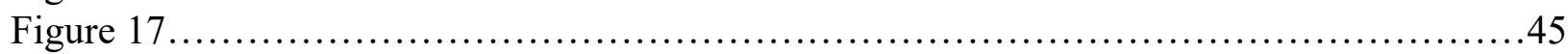

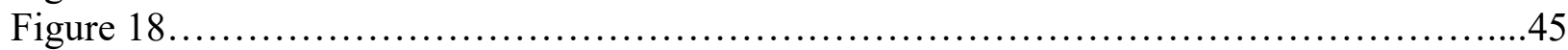

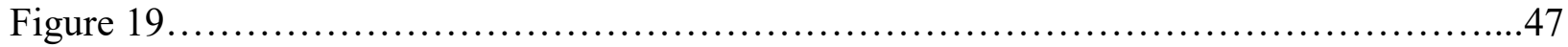

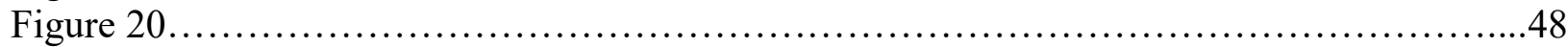

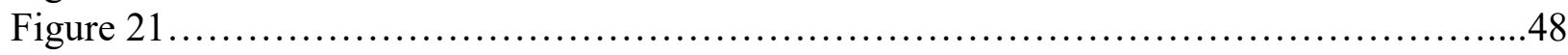

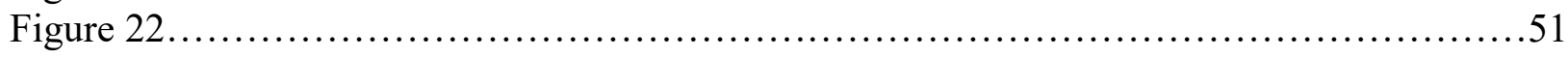

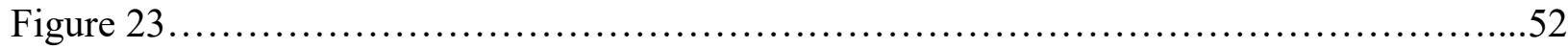




\section{LIST OF APPENDICES}

Appendix A: News Sources ........................................................5

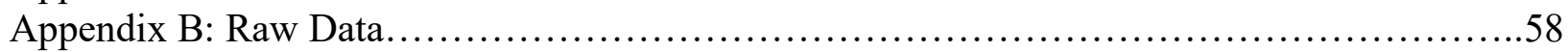

Appendix C: Meme References................................................6 61 


\section{INTRODUCTION}

As the internet continues to evolve, internet memes are becoming an increasingly popular method of quick and easy online communication. Memes are commonly known as "an amusing or interesting item (such as a captioned picture or video) or genre of items that is spread widely online especially through social media” (Merriam Webster's Collegiate Dictionary, 2020). Routinely, memes go viral and are distributed on social networking platforms because they offer the potential for high user engagement. Memes provide snippets of humour and wit, while also often responding to larger socio-political issues and concerns. The value of the consumption of internet memes derives from their ability to communicate easily digestible information in a memorable, visually stimulating, and often humourous way. Their often light-hearted tone contributes to people's ability to comfortably consume information in a world filled with negative headlines and heated online debates. Memes provide a necessary break in tension and have the potential to effectively engage their audience with social commentary. This paper will examine memes as a distinct method of digital communication. More specifically, I will analyze the use of memes during the COVID-19 crisis. I will be examining whether memes provide a comforting and relatable medium for dealing with public anxieties and for communicating complex issues to large audiences. I will also be analyzing how data on the popularity of memes can provide a better understanding of their communicative limits and potential. My primary focus of this paper will be whether memes are a valuable communicative tool that younger generations can use to engage with current events and whether memes can offer a viable alternative to mainstream media and news outlets. As teens and young adults comprise a large 
proportion of active social media users, identifying the messages and meaning of memes is crucial for cultural analysis of today's modes of digital communication.

The literature review will focus on works about the consumption of memes, meme usage as a coping mechanism, the relation of memes to mainstream media, and the emergence of often unheard voices that can result from this new form of communication. Following the literature review, I present my research questions, methodology as well as my findings and discussion. Findings from this paper will inform future communicative research related to youth engagement with current events and online tools. Understanding these findings will aid in building new communication strategies that appeal to a younger demographic and promote new and emerging online voices. Building communicative strategies based on online habits of interaction can assist in creating impactful social changes in the future. Considering the often unheard voices during a crisis may also lead to further inclusive changes to policies and societal behaviours.

\section{LITERATURE REVIEW}

\section{Media Consumption in Relation to Memes}

Memes have become a popular form of online media due to their instantaneous and interactive nature. In Horváth and Mitev's study of meme popularity and its cultural contexts, they compare the emergence and usage of memes to a meteor, or a "memeteor", due to the speed, scope and almost immediate impact internet memes can have on their audiences (Horváth and Mitev, 2016). The affordances of social media creates an environment where sharing memes to a personal network is only a click away. The scholars also observe memes to be "enriched by humorous content, societal questions, emotionally involving situations, and potential for self-expression" (Horváth and Mitev, 2016). The element of humour often associated with 
memes, while often sparking more thoughtful conversation, contributes to their virality across open channels such as social media as well as within more private virtual conversations.

Research by Grant Kien (2013) in Media Memes and Prosumerist Ethics suggests that there are four primary ways that audiences use media texts. These include entertainment, social interaction, personal identity formation and information (Kien, 2013). Kein further theorizes that in our era of digital social media, it is entirely possible for memes to be used in all four of these ways simultaneously (Kien, 2013). It is their ability to be multiple things at once that makes internet memes an increasingly powerful mode of communication.

The viral media and discussions that evolve from internet memes allows them to be examined as "artifacts of participatory digital culture" (Wiggins and Bowers, 2015, p.6). This is due to the fact that popular memes evolve into "meme templates" that encourage "a genre development featuring stages of maintenance, elaboration, and modification" (Wiggins and Bowers, 2015, p.11). The creation of a genre surrounding one "meme template" encourages more media consumption through the formation of new internet memes often relating to the original topic, further seducing individuals to become engaged with a given topic or conversation. The continuous cyclical response that internet memes generate before losing relevance creates an active and evolving virtual space of conversation, creation and connection.

\section{Memes as a Coping Mechanism}

Using images to make sense of social phenomena is not unique to the digital age. For example, communicating anxieties about disease and death through "viral" imagery can be dated back to 15th-century England with the emergence of the "Grim Reaper" character (Marcus and Singer, 2017). Oftentimes, images that make anxieties visible exist to ease tensions through 
comfort and creating visuals serves as a tool for bridging the gap between internal unease and the social world. However, "the simplistic explanation that jokes inevitably 'rise to the occasion to articulate anxieties' glosses over the different ways in which humor is an important social response and how dominant social forces both shape and reject it" (Marcus and Singer 2017, p.349). Marcus and Singer analyze how imagery can be used to combat fear and suggest that representations displayed in mainstream media allow people to regain agency over a given set of circumstances. More recently, for example, the emergence of an illustration depicting the Ebola outbreak named "Ebola-chan" not only reduced fear surrounding the disease, but also decreased anxieties about participating in discourse surrounding the topic (Marcus and Singer, 2017). By reclaiming agency over the imagery related to a serious or frightening topic, participants were able to manage frightening situations with a bit of humour, ultimately obtaining a greater sense of comfort. This phenomenon can be regarded as a recurring necessity through the example of "Corona-chan" that has emerged due to COVID-19. The creation of this character has, as it was with the Grim Reaper, once again created a "puppet" that can be used to ease anxieties surrounding complex discourse.

Similar findings were presented in Yang's look at the Brussels, Belgium security lockdown in 2015 that occurred due to the threat of a terrorist attack (Yang, 2016). During the lockdown period, citizens took to Twitter to update one another about the situation. Authorities soon ordered them to stop using the hashtag \#Brusselslockdown to update users because terrorists had access to the Twitter information as well. Soon after, the hashtag transformed to share images and memes of kittens to scramble the messaging (Yang, 2016). Through examining the trend and interviewing participants, however, Yang categorized participants using the 
hashtag into three distinct categories. These included the "Political Sharer" who used the format of kitten memes to dilute results for terrorists, the "Apolitical Sharer" who primarily used the platform as a creative release and humour, and the "Conversationalist" who used the meme format to instigate larger discussions about the current socio-political climate (Yang, 2016). These findings suggested that despite the discrepancies of the motivations to share using \#Brusselslockdown, all user types were able to come together to create a safer virtual space of support in a time of physical isolation and collective fear and anxiety.

With this in mind, it is also important to acknowledge the potential dangers that can arise from meme-generated communal connection. For example, during times of crisis or heightened anxieties such as those mentioned above, participants may choose "content that fits with the mood they are in at that moment, as they strive to maintain their state of excitation" (Kien, 2019, p.54). In other words, if individuals are distressed, they may continue to seek out distressing information and memes that have the potential to create mass hysteria (Kien, 2019). Additionally, smaller communities online that provide comfort may also harvest "a mentality of groupthink, and not be open to challenges or influences that might under normal circumstances improve the strength of their community and new intellectual breakthroughs" (Kien, 2013, p.556). The creation of narrow minded content and communities may hinder the productivity of online discourse, and can only be recognized if online participants are critically thinking about their habits of consumption. However, similar groupthink mentalities are present within mass media channels. When used effectively online communities and the usage of internet memes have the ability to combat mainstream perspectives with fresh discourses surrounding any given subject. 


\section{Memes vs. Mainstream Media}

Although internet memes have a high turnover rate in terms of what becomes and remains viral, research indicates that the popularity of memes and the longevity of their fame may correlate with what is reported in "traditional media and real-world events" (Weng et al., 2012, p.4). Feeding off of the 24-hour news cycle and what mainstream media deems noteworthy, internet memes provide an outlet that allows users to react and combat conventional or more orthodox discourses. Humorous memes and, for example, "RIP trolling", where individuals make jokes on facebook memorial pages, can be seen as more than merely providing shock value as they push back "against a corporate media environment that fetishizes, sensationalizes, and commoditizes tragedy" (Marcus and Singer, 2017, p.349). Rallying against a current issue in this way opens up new perspectives on current events. This idea is echoed by a study conducted by Literat and van den Berg (2019) on a popular Reddit subreddit titled "r/memeeconomy". The community uses wall street style jargon to rate and "invest" in upcoming memes based on their perceived set of success criteria. Results from this study indicate that a major factor "identified by meme traders as significantly impacting a meme's value is its topicality or cultural relevance" (Literat and van den Berg, 2019). In addition to the topical and cultural relevance of a meme, findings also suggest that "the most significant determinant of value appears to be the positioning of the meme in relation to mainstream Internet culture: memes need to be popular enough to gain traction, but not too popular to become mainstream and be adopted by normies and become a 'normie meme"' (Literat and van den Berg 2019). This specific criteria highlights that the ability to create new public discourse surrounding a topic using less traditional perspectives is what results in a meme growing in popularity. 
Memes provide users with an ability to popularize what could otherwise be seen as unpopular or edgy opinions. The usage of internet memes therefore encourages new and alternative voices to join larger socio-political conversations (unless these voices are cancelled -- i.e. censored -- by the meme platforms themselves, as has been seen recently with Twitter banning thousands of users whose posts were deemed unpalatable to current sensibilities) (Collins \& Zadrozny, 2020).

\section{Memes and the Emergence of Unheard Voices}

Internet memes provide a channel of communication that not only has the ability to give a voice to unpopular opinions, but also to bring together the voices of the unheard. The usage of memes is found predominantly amongst the Millenial and Gen $\mathrm{Z}$ generations. This is due to the fact that these generations were raised within the digital sphere from a very young age. As a result, they are more familiar with the codes of communication utilized in creating and responding to viral memes (Kien, 2013). Grundlingh (2018) studies the similarities of memes and speech acts. By comparing memes to other conventional speech acts, Grundlingh suggests "an individual posting a meme as a comment or just posting memes in general, must assume that the addressee shares the same codes and that he/she will interpret the meme in a specific way before choosing which meme to use" (Grundlingh, 2018, p.157). In doing so, memes unlock unique ways of interpreting phenomena based on hidden references that may be lost to audiences outside of the initially intended community. As mainstream media can be argued to be monopolized by an older or more educated faction who are in charge of disseminating information, internet memes serve as an alternative to traditional narratives. The emergence of new speech acts in the form of memes does not only allow for alternative discourses that offer 
new perspectives, but can also awaken "armchair activism” (Kien, 2013). As Kien notes, for example, "anyone on the Net has the power to affect stock prices" (Kien, 2013, p.554).

Obtaining this new capability, unheard voices have a definitive reason to utilize memes for socio-political commentary and influence.

\section{RESEARCH QUESTIONS}

Building on the literature review, my research will be focused on socio-political memes and commentary related to the economy, government and social inequalities that have emerged during the COVID-19 pandemic. This paper will be driven by the following three research questions:

1. What is the relationship between memes and mainstream media?

2. How do memes provide a channel for understanding current events in the face of more dominant and mainstream discourses?

3. Do memes empower younger demographics to critically engage with current events? Findings from these research questions will attempt to fill gaps within current scholarly data on memes. A greater in depth analysis of the communicative power memes hold within our current social world can provide insight into upcoming shifts within the realm of communications.

\section{METHODOLOGY}

A mixed methods approach to data collection will be used to understand the relationship between memes, mainstream media and the audiences consuming this form of communication. As the literature review conveys, a meme can be examined as a speech act. Therefore, an effective way to investigate its complex relationship with traditional mainstream media is by 
examining a variety of perspectives during the data collection process. In doing so, the final outcome of research becomes "stronger than either method individually" while allowing for the exploration of "complex aspects and relations of the human and social world" (Malina and Selto, 2011, p.49). Following a mixed methods approach also provides greater precision within findings, as the risk of biased interpretation decreases with the combination of numerical and contextual data. Using this approach, memes within two social media platforms, Instagram and Reddit, will be evaluated using quantitative analysis, content analysis and critical discourse analysis.

\section{Quantitative Analysis}

Quantitative analysis will primarily be used to examine Instagram's metrics. This will be completed by reviewing data involving the number of likes and comments for each post analyzed. For every observed Instagram page, I will analyze the average number of likes and comments for specified periods of time and compare the data to metrics for memes surrounding COVID-19. Gathering quantitative data in this manner will provide insight on a potential relationship between current events and the higher engagement of related memes. These findings will establish a numerical foundation that will be further explored using a qualitative lens, in the form of a content analysis.

Quantitative analysis will not be used while exploring memes within my second social media platform, Reddit, as the data set that I will be exploring is much smaller. Additionally, all memes within the subreddit, a smaller channel within Reddit, that will be assessed relate to COVID-19. As such, an analysis of the correlation between current events and engagements 
would not be possible. Instead, closer analysis through a qualitative approach will be more effective.

\section{Qualitative Analysis: Content Analysis}

Content analysis will be an important tool for the examination of the social media platforms Instagram and Reddit. The use of this method will assist in examining underlying themes in memes with high engagement. Using a content analysis approach is beneficial for a large volume of memes due to the consistency that coding can offer. For this paper, I will begin by using open coding. I will manually analyze each meme individually to identify major recurring themes. The chosen coded themes will be consistently revisited with "constant self-scrutiny, reflexivity and the analysis of any conclusions [drawn]" (Blair, 2015, p.19). This is to ensure all possible outcomes are considered while removing personal bias during the coding process. Using the foundation open coding provides, I will then proceed to use axial coding to develop a broader understanding of the frequency of coded themes as well as the relationship between major underlying themes. Recording these codes on a spreadsheet will assist in further manipulation of data moving forward. Greater detail on how this will be accomplished will be explained in the sampling portion of this paper.

Using a content analysis approach while examining memes on Instagram will assist in reinforcing or challenging existing quantitative data. Doing so will help draw well rounded and comprehensive conclusions about memes, audience engagement and larger social discourses taking place in current events. Similarly, a content analysis while exploring Reddit will provide insight on the community's behaviours of engagement and interests before delving into a critical discourse analysis of the comments and interactions taking place on the platform. 


\section{Critical Discourse Analysis}

Critical discourse analysis will be used to examine comments made on posts of memes surrounding COVID-19 on Reddit. This method was chosen to highlight how comments made about memes on current events "make a significant and specific contribution to critical social and political analyses" by providing "an account of the role of language, language use, discourse or communicative events in the (re)production of dominance and inequality" (Van Dijk, 1993, p.253). By examining how current events are discussed on the platform, I will be able to analyze whether comments provide meaningful social commentary about current events. As Fairclough states, critical discourse analysis

"brings the critical tradition in social analysis into language studies, and contributes to critical social analysis a particular focus on discourse, and on relations between discourse and other social elements" (Fairclough, 2013, p.178).

In a broader sense, this method will provide greater insight on whether memes and the platforms promoting these memes provide a safe space for youth engagement and discussions that go beyond humour. A critical discourse analysis in this manner in addition to a content analysis of major underlying themes within the comments will give insight on the quality of discussions occurring and their potential for larger-scale societal impact.

\section{Selected Social Media Platforms for Analysis}

The platforms that were selected for analysis were Instagram and Reddit. Other platforms such as Twitter, Facebook and Tiktok were not selected due to user algorithms playing a larger role in dictating an individual's media engagement and consumption. In contrast, both Instagram and Reddit use dedicated community focused pages that users are able to visit and follow to consume content in a clearly defined space. Additionally, many of these accounts are initially 
privatized until granted permission to follow, providing an added sense of privacy, exclusivity and community. This differentiation also allows for a more accurate analysis of user engagement of individuals seeking out memes. Those who follow meme pages are also familiar with the specific nuances memes are able to encompass. The audience being analyzed is therefore individuals actively turning to memes as a form of communication themselves.

Instagram was selected due to the prevalence of dedicated meme accounts that the platform hosts. Accounts are well known for consolidating popular content from numerous other platforms as well as for providing original content to their audience. Oftentimes, these accounts are privatized to promote a strong following and to compete with other largely followed accounts in the space. For the purpose of this paper, four popular meme accounts were selected to be analyzed. Each account that was selected has at least 2.5 million followers, with some accounts catering to up to 15 million followers. For each account, I will be looking at popular news stories in North America and examining its impact and engagement within the chosen Instagram meme accounts. More specifically, I will be collecting data on the number of posts related to the news stories as well as the number of likes and comments the posts receive compared to the average metrics of the page. The selection of news stories and meme accounts will be explored further in the subsequent sampling section of this paper. The collected data will provide an indication of whether there is a strong relationship between mainstream media news stories and daily memes. It will also give insight on the audience's communicative behaviours and engagement with current events. The methods of analysis that will be used for this platform include quantitative analysis of engagement metrics, and a content analysis of the most liked and commented memes observed during the days of data collection. 
Reddit was also selected as a social media platform for analysis. As an online content sharing platform, Reddit provides a quick and easy way to find both viral and niche content based on user interest. The platform is "organized around communities of interest (subreddits) which are moderated by volunteer users" (Literat, 2019). Users are able to actively choose the communities they would like to interact with as well as discover new subreddits through browsing Reddit's "front page". The platform is also known to be "the site of origin for content that goes viral through sites such as Facebook and Buzzfeed" (Literat, 2019). For the purpose of this research study, I will be using the platform to specifically look at the subreddit "r/coronavirusmemes", which has over ninety thousand active members. Based on the description given by its moderators, this subreddit describes itself as a community that is "getting a laugh out of Coronavirus while [they] still can, and spreading happiness in a time of distress" ( $\mathrm{r} /$ coronavirusmemes, 2020). Analyzing a niche community online that creates and shares content about COVID-19 will provide insight into whether memes provide a channel for larger discourse about current events, and if so, what the engagement looks like. To obtain these findings, I will be using the subreddit's sorting function to analyze the top five posts of all time. With the top five memes, I will first conduct a content analysis of the memes themselves. Following the content analysis, a critical discourse analysis of the comments under each meme will be conducted to determine whether the meme platform provides an opportunity for larger social discussions, and if so, determining how exactly this occurs. Further information on the specifics of the analysis will be discussed in the subsequent sampling section of this paper.

\section{SAMPLING}

Instagram: Choosing Meme Accounts 
As introduced within the methodology section of this paper, the platform Instagram will be examined through four viral meme accounts, each with a following of at least 2.5 million users. Each account will be analyzed to understand the relationship between the engagement of memes and current events, and whether the chosen accounts take advantage of topics discussed within mainstream media. The accounts that will be used to conduct this study are as follows:

\section{Memes.com}

This account has a following of 6.5 million users with over 14 thousand posts. The account posts frequently, with an average of over 7 posts a day.

\section{Daquan}

This account has the largest following of the meme accounts analyzed, with 15.2 million followers and over 8 thousand posts. The account's frequency of posting is low, with an average of only 2 to 3 posts a day. However, this account has the highest engagement of memes. The account strongly values the quality of its memes over quantity. It is also important to note that this account will remove posts that do not meet engagement standards, as some of the memes that will be analyzed have since been removed.

\section{Kalesalad}

This account has a following of 3.9 million users and over one thousand posts. The accounts acts as a general meme account, as well as several niche accounts branching from the brand including "Kalesaladsports", and location specific accounts such as "KalesaladNY." The account "Kalesalad" was chosen to be analyzed due to its mass appeal to a wider audience. The account posts frequently on the days that will be examined, with an average number of 6 posts a day. 


\section{Thefunnyintrovert}

This account has 2.5 million followers with over 6 thousand posts. The account posts frequently on the days that will be examined, with an average of 4 posts a day.

For each Instagram page, memes corresponding with the dates each news story takes place will be analyzed. As meme accounts often repost original content found on other platforms, I acknowledge that there may be a delay between posts and current events. To account for this delay, posts are analyzed the day a news story breaks as well as the day after to collect more accurate data. These findings will be recorded using a data collection template that will be discussed in further detail following this subsection. All meme accounts were reached out to after the selection process in an attempt to obtain insight on the demographics of their user base that they would have access to. However, I did not receive a response from the accounts.

\section{Instagram: Choosing Media Stories}

For this paper, the selection of news stories examined to compare the relevance of current events to meme pages will be concentrated within North America. The selected stories will be To determine the five news stories surrounding COVID-19 within North America, three primary resources were considered. The first resource is from the official website of the World Health Organization (WHO). The website provides a timeline of events of how the pandemic unfolded, as well as major world events surrounding COVID-19 since its origin. Due to the fact that the research for this paper is focused on North American stories, WHO's presented timeline of events was cross referenced with two localized timelines. One was presented by NBC News, an 
American news network. The other timeline was presented by the Canadian Broadcasting Corporation (CBC). Both works focused on major news stories surrounding Canada and the United States (See Appendix A). From these resources, major news stories were selected based on the impact it would have on a global scale, as well as stories younger demographics are directly impacted by. The news stories that were selected are:

1. March 11, 2020: WHO Officially Declares COVID-19 as a Pandemic

2. March 13, 2020: Canada Closes Borders and March 16, 2020: US Closes Borders

3. March 24, 2020: Summer Olympics postponed until 2021

4. March 12, 2020: Canadian School Closures and March 16, 2020: US School Closure

5. March 31, 2020: Wall Street Ends one of the Worst Quarters in Stock Market History News stories were chosen to all occur in March 2020, since numerous policy changes and implementations to adjust to the pandemic occurred within this time period. The abundance of new information and implementations resulted in a higher state of global panic surrounding COVID-19 during this timeframe. Therefore, analyzing news stories and their relationship to memes during the height of adjustments and anxiety will display a stronger impact between the relationship of mainstream media and memes. Although there is a slight overlap of dates within some periods of analysis, the diversity of topics in the selected news stories will provide a unique opportunity to observe which stories engage younger demographics the most.

Instagram: Quantitative Analysis

Data collection for quantitative analysis will be separated by the five major news stories to be examined. To better understand the behaviours of each Instagram page, observations for 
every account will also be separated. The recorded data will include the number of postings within a two day span of each news story, and the number of likes and comments each meme received. It is important to note that the number of likes and comments are prone to increase daily. To keep my data consistent, all data surrounding a news story will be collected on the same day, approximately one month after its initial posting. Since memes will typically receive the most engagement within the first week it is posted on an account, the gap between the date of its posting and data collection will display an accurate representation of its popularity. This data will be recorded on a spreadsheet as shown in Figure 1. For full access to the raw recorded data as seen in Figure 1, see Appendix B. Upon this initial compilation of data, the mean, median, and mode will be calculated for each subcategory. These results will provide a foundation for the types of engagement that can be typically observed, before delving into more specific analysis on the types of memes that receive the highest engagement.

WHO officlally declares tovil]- 19 as a pandemic

\begin{tabular}{|c|c|c|c|c|c|c|c|c|c|c|c|c|c|}
\hline \multicolumn{4}{|c|}{ Homeracam } & \multicolumn{3}{|c|}{ Doquan } & \multicolumn{3}{|c|}{ Kalesoled } & \multicolumn{4}{|c|}{ Thetunnyintravert } \\
\hline \multirow[t]{2}{*}{$r=0$} & \multicolumn{2}{|c|}{ oflan } & adcerres & \multirow[t]{2}{*}{ ror: } & - vilaner. & \multirow{2}{*}{ - decers } & \multirow[t]{2}{*}{ Per: } & min & xtomers & \multirow[t]{2}{*}{ Nen: } & \multicolumn{2}{|c|}{ txis } & iscoments \\
\hline & , & 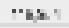 & $\cdots$ & & sest & & & . & 2 & & , & 2.20 & sz \\
\hline & $=$ & uns & 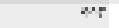 & $?$ & wי & $v r$ & $=$ & 2er & $\mathrm{x}$ & & , & ave & wh \\
\hline & s & 125 & s.t & , & 120 & d.a & J & ows & 2 & & 2 & $+1 / 2$ & $\approx$ \\
\hline & - & 170,0 & $\mu F$ & c & $\infty$ & ve & 4 & $\cos$ & $\therefore$ & & 4 & $4 \pi$ & $=x$ \\
\hline & . & ines & $6 .$. & $s$ & as.a & x.e. & $s$ & $x+3$ & in & & $s$ & $w / 2$ & $\pi$ \\
\hline & 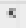 & $1,0.6$ & $1 \times$ & s & 1000 & מa & - & 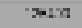 & 100 & & - & $20 \cdot 2$ & ai \\
\hline & $i$ & $" h \mathrm{Y} !$ & $4: 2$ & $i$ & ixiti & 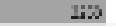 & 8 & האירי & rs & & ? & $t \cdot x$ & $x$ \\
\hline & 5 & $103 \times 3$ & s.t & & & & 8 & Exod & in & & 2 & 210 & .6 \\
\hline & 3 & 13.tie & $x=$ & & & & 3 & $20: 0$ & 10 & & 5 & $\sin x$ & $*$ \\
\hline & 1) & $w \geqslant 0$ & $\therefore 4$ & & & & 3 & $\cdots$ & $\omega$ & & 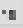 & $n \rightarrow \infty$ & 100 \\
\hline & 1 & 125 & $m$ & & & & +1 & $a \cdot x$ & 31 & & " & $r v$ & $\%$ \\
\hline & $\mathbf{u}$ & Ien. & sta & & & & $\cdot 2$ & $2 \mathrm{Lt} \cdot 2$ & in & & & & \\
\hline & is & issert & u=? & & & & 18 & $2=1$ & $\mathrm{ki}$ & & & & \\
\hline & 1 & 1.4.1.2. & 2.2 & & & & -4 & ma & $*_{4}$ & & & & \\
\hline \multirow{2}{*}{\multicolumn{2}{|c|}{ is }} & $\mathrm{k}, \mathrm{w}$ & 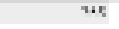 & & & & 5 & 2.: & we & & & & \\
\hline & & & & & & & 16 & s.is & $\omega$ & & & & \\
\hline Ua: & & ieu: & $w$ & Yus & гн:мхх & 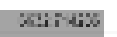 & Wue & Ge: 18 & $20: 18$ & Twe & & stisac:ix & 2L Xx:20 \\
\hline$\omega \approx$ & & $10 y=8$ & 20.4 & $y=$. & 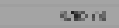 & $2 \times 4$ & $n=1$ & Trwa & $\tan t$ & $n+4$. & & ats & a \\
\hline \multirow[t]{2}{*}{ Ucsin } & & at: & $<: 7$ & Nost & Nhit & niue & Vout & $m$ & $\cdots$ & $=\times 1$ & & $\infty$ & 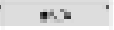 \\
\hline & & & & Nine : : & E: 421 & at a a & & & & & & & \\
\hline
\end{tabular}

Figure 1 
In addition to this data collection process, memes relevant to the news story and other aspects of COVID-19 will be coded and recorded separately with the corresponding engagement metrics. The data collection process for memes related to current events is illustrated in Figure 2. For full access to the raw recorded data as seen in Figure 2, see Appendix B. Recording memes specific to current events separately serves as an accessible method of quick comparison between average metrics within a two day span on any Instagram account compared to a meme that is story specific. Having data formulated in this manner will also provide a channel for comparing how many of the memes recorded were about current events within the data set. Findings can then be easily displayed using graphing functions the spreadsheet used for data collection offers.

\begin{tabular}{|c|c|c|c|c|c|c|}
\hline $\begin{array}{l}\text { Account } \\
\text { Nante }\end{array}$ & News Topic & $\begin{array}{c}\text { \# of } \\
\text { Correlating } \\
\text { Posts } \\
\text { (2 Day span) }\end{array}$ & $\begin{array}{c}\text { \# of } \\
\text { covtD-19 } \\
\text { Mennes }\end{array}$ & \# af I.ilces & $\begin{array}{c}\text { \# of } \\
\text { Comments }\end{array}$ & $\begin{array}{l}\text { Average } \\
\text { Metrics } \\
\text { (2 Day Span) }\end{array}$ \\
\hline $\begin{array}{l}\text { thefunnyi } \\
\text { ntrowert }\end{array}$ & 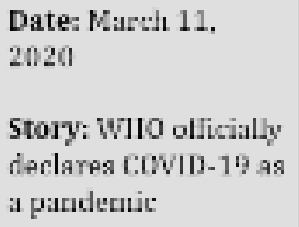 & $\begin{array}{c}8 \\
\text { vicotco } \\
\text { viary }\end{array}$ & $\begin{array}{l}3 \\
\text { 'Tom Hianles } \\
\text { Turkmy } \\
\text { Bourater } \\
\text { Rerauver }\end{array}$ & $\begin{array}{l}42,705 \\
70,007 \\
\\
66,612 \\
37,032 \\
116,307\end{array}$ & $\begin{array}{l}140 \\
365 \\
\\
341 \\
156 \\
1,363\end{array}$ & 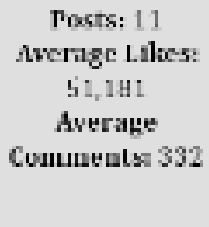 \\
\hline $\begin{array}{c}\text { mpmeseo } \\
\text { I }\end{array}$ & $\begin{array}{l}\text { Dale: March 11, } \\
2020 \\
\text { Sury: WH0 officially } \\
\text { teclares COVID-19 as } \\
\text { a pandamis: }\end{array}$ & $\begin{array}{c}2 \\
\text { Thue Simpsous } \\
\text { "Drush yo } \\
\text { Teeth" }\end{array}$ & $\begin{array}{l}3 \\
\text { Fligits so } \\
\text { Cheap } \\
\text { Cheap } \\
\text { Fights }\end{array}$ & $\begin{array}{l}196,581 \\
96,781 \\
125,676 \\
1001,147\end{array}$ & $\begin{array}{l}1,918 \\
756 \\
1,011 \\
5151\end{array}$ & $\begin{array}{c}\text { Posist } 15 \\
\text { Average I.fikes: } \\
124.234 \\
\text { Average } \\
\text { Comments: } 946\end{array}$ \\
\hline Dąquan & $\begin{array}{l}\text { Date: March } 11 \text {, } \\
2020\end{array}$ & 2 & 4 & & & $\begin{array}{c}\text { Posts: ? } \\
\text { Average Lakest }\end{array}$ \\
\hline
\end{tabular}

Figure 2

Instagram: Qualitative Content Analysis

After conducting a quantitative analysis, the most liked and most commented posts from each page on every date analyzed will be compiled for a qualitative content analysis. This 
method of analysis will be used to identify major themes occurring within the most popular memes observed. Findings from this method will provide insight into the hidden elements of success when viewing memes and their relationship memes have with mainstream media. Of the compiled list of most liked and commented memes, three memes were removed from their meme pages between the time quantitative data was collected to when a content analysis began. As I was unable to contact the account holders of the meme accounts, it is unknown why they were removed. The three memes will remain in the quantitative research already conducted, but will be removed for the content analysis portion of this paper. The initial list of memes analyzed totalled 39 memes. Due to slight overlaps in the dates of news stories, 10 of the most liked and commented memes were repeated. All repeating memes were removed from the content analysis, leaving 29 unique memes to be examined.

From the compiled list of memes, open coding was initially conducted. After manually coding each meme, nine major themes were identified to hold significance. The identified themes are the following:

\section{Theme 1: Pop Culture Reference}

Memes utilizing existing well known references to TV shows, movies, music, games and other news stories to create a more witty, relevant and timely meme.

\section{Theme 2: Adds Onto an Existing Online Trend}

Memes following familiar meme templates or trends to gain further traction and versatility of the trend's usage.

\section{Theme 3: Works with a Mainstream Media Story}


Memes that directly comment on current events and relevant social issues currently taking place when a post is made.

\section{Theme 4: Funny}

Memes with the primary focus of making its audience laugh. This is often conducted in the form of a witty remark or a funny video reshared with an intriguing caption.

\section{Theme 5: Distraction}

Memes with the primary intention of distracting its audience from heavier topics, often occur at the height of a current event.

\section{Theme 6: Generation Specific Meme}

Memes that make reference to an item or event that is more specific to a younger generation and may not be understood by older generations.

\section{Theme 7: Opens Discourse}

Memes with the intent to open discussion through either posing a question, opinion or commenting on a relevant topic.

\section{Theme 8: Social Commentary}

Memes that address current events or comment on a social phenomenon. Often associated with opening discourse on a particular topic or sharing an opinion.

\section{Theme 9: Coping}

Memes with the primary agenda to show its audience they are not alone or provide helpful tips and resources for dealing with mental health related issues during a time of crisis. 
The 29 unique memes will then be coded using the axial coding technique to determine the number of times each theme occurred. There are no limitations to how many of the themes a single meme may fall under. The method of how this is recorded is shown in Figure 3. Figure 3 displays the coding chart for the most liked memes, but identical measures were taken for the most commented memes as well. The most commented memes also measure the same themes listed above. To access the full data on coding, see Appendix B for details. Data collected from the content analysis will then be ready to be employed to identify key findings about the significant underlying factors memes carry in current circumstances.

\begin{tabular}{|c|c|c|c|c|c|c|c|c|c|}
\hline Meme & \multicolumn{2}{|c|}{ Theme 1: 1 Thene 2: } & \multicolumn{3}{|c|}{ Thene 3: 'Theme 4; ] Theme 5; } & \multicolumn{2}{|c|}{ Theme $6 ;$; Theme 7 ; } & \multicolumn{2}{|c|}{ Theme 8; Thene 9; } \\
\hline Simpeons Prediction & 1 & 1 & 1 & & & & & & \\
\hline Bcomer Remover & 1 & 1 & 1 & 1 & & 1 & & & \\
\hline WHO let the dogs out & 1 & & 1 & 1 & & & & & \\
\hline Fovourica Person & & & & & & & 1 & & 1 \\
\hline Titaric & 1 & & 1 & 1 & & 1 & & & 1 \\
\hline Fight Over Eeans & & & 1 & 1 & & & 1 & 1 & 1 \\
\hline Kerrye Face & 1 & & 1 & 1 & & & & & 1 \\
\hline CSFN Chers & & & 1 & 1 & & & & 1 & \\
\hline Duy asn lesisnd & & & 1 & 1 & & & & & \\
\hline Prafresent Fisl & & & & 1 & & 1 & 1 & & 1 \\
\hline Gand Nerts: & & & & 1 & 1 & & & & 1 \\
\hline Hugged loghter & & & & & & & & & 1 \\
\hline Ging Cistt & & & & 1 & & & & & 1 \\
\hline Boytriand's Job & & & 1 & & & & 1 & 1 & \\
\hline Uno Government & & 1 & 1 & 1 & & 1 & 1 & 1 & \\
\hline Trylor Swift & 1 & & & 1 & & & & & 1 \\
\hline Can' Spend the Ngint & & & & & 1 & & & & 1 \\
\hline 2020 Planner & & & & 1 & & & & & 1 \\
\hline
\end{tabular}

Figure 3

Reddit: Content Analysis

Similarly, a content analysis will be conducted for the five most popular memes on the subreddit " $r$ /coronavirusmemes". These memes will be identified using the function to filter the 
posts with the most "upvotes" of all time on the subreddit. A smaller sample size will be chosen for this platform in order to perform a more in depth analysis of the hidden themes identified on the subreddit. The five most popular memes will be analyzed manually. Findings from this analysis will be used to identify similarities and differences between memes on two different platforms, while simultaneously attempting to gain a broader understanding of the relationship mainstream media plays in the formation of memes. As all the memes on the subreddit are related to the specifics of COVID-19, it is crucial to use this space to understand which news stories in particular, if any, catch the attention of its audience.

In addition to a content analysis of the most popular memes on "r/coronavirusmemes", a content analysis on the comments for each of these memes will also be conducted. Before jumping into a critical discourse analysis of the comments, coding major themes found will help identify the types of discourse that are present. Comments were separated by post and sorted by the most to least upvoted comments. After using an open coding process, 6 major themes were determined to be the most prevalent. The themes are as follows:

\section{Theme 1: Pop Culture Reference}

Utilizing existing well known references to TV shows, movies, music, games and other news stories to create a more witty, relevant and timely comment.

\section{Theme 2: Political/Social Commentary}

Comments that point to current events or comment on a relevant social issue.

\section{Theme 3: Funny}

Comments with the primary focus of making their audience laugh.

\section{Theme 4: Off Topic}


Comments that do not significantly relate to the discussion of the meme or topic in any way.

\section{Theme 5: Asking a Question}

Comments that ask questions or clarifications about current social issues

\section{Theme 6: Coping}

Comments using the platform to share their experiences, providing advice or emotionally connecting to the meme in some way.

Comments within each post will then be recorded to display the frequency of prevalent themes as seen in Figure 4. Figure 4 looks at how data will be collected for the first post analyzed, but the identical method was used for each post observed. For the full coded data, see Appendix B.

\begin{tabular}{|c|c|c|c|c|c|}
\hline & & & RELDIT & COMIEN & TS PusL \\
\hline Canren & 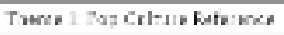 & 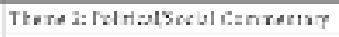 & There Zetrers & Twoe 4 ott Tepiz & There? Aoting a Cue on Tarus ceping \\
\hline In thorgutidas & 1 & & & & \\
\hline b.xini:1.12. & 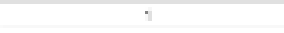 & & & & \\
\hline 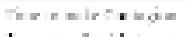 & $?$ & & & & \\
\hline Cespestrong & 1 & 1 & & & \\
\hline 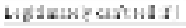 & & & & & 1 \\
\hline 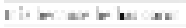 & & : & & & \\
\hline$a+16$ & & & & ? & \\
\hline 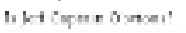 & & & & 3 & \\
\hline WAXWGIL & & 1 & & & \\
\hline 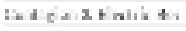 & ? & & 1 & & \\
\hline 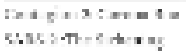 & & & $\vdots$ & & \\
\hline zas $x 11: 4 x$ atr:s & & 1 & & & 1 \\
\hline 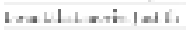 & & & & & 1 \\
\hline resdaniditidi & & & i & & \\
\hline
\end{tabular}

Figure 4

\section{Reddit: Critical Discourse Analysis}

Following a broad examination of the comment sections within the top five memes of all time posted on r/coronavirusmemes, an in depth review using critical discourse analysis will be conducted. The primary approach I will be implementing within my critical discourse analysis is interdiscursivity. This is defined as "a mixing of diverse genres, discourses, or styles associated with institutional and social meanings in a single text" (Wu, 2011, p. 96). As my findings will be 
stemming from the comment section of meme posting often consisting of a variety of referential statements and opinions, interdiscursivity provides a suitable conceptual framework for analysis. Under each meme, data will be sorted from the most popular comment, which is determined through the number of "upvotes" each comment receives, to the least popular comment. Each comment will be sorted as displayed in Figure 5. The critical discourse analysis will be compared and contrasted with data established within the content analysis of the comments to observe how discussions surrounding current events are handled in what is meant to be a safe and easy going environment. An interdiscursivity lens will also illuminate if and how examined discourses within the comment section may instigate social change. All analyses will be completed manually and displayed in greater detail within the Findings section of this paper.

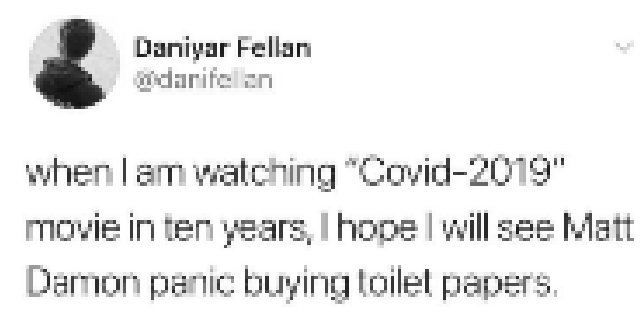

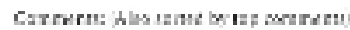

\begin{tabular}{|c|}
\hline 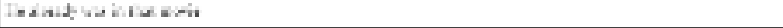 \\
\hline 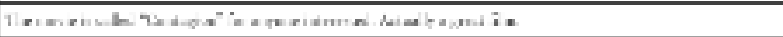 \\
\hline $1.0 .=60: 0=19$ \\
\hline 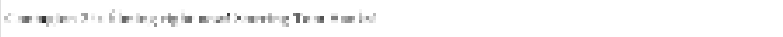 \\
\hline 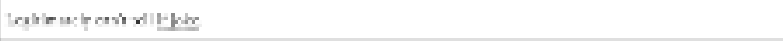 \\
\hline 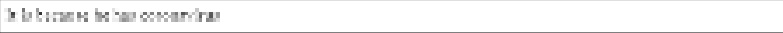 \\
\hline Giwn'unt \\
\hline hingunatina? \\
\hline$\theta=\operatorname{lin} d=4$ \\
\hline 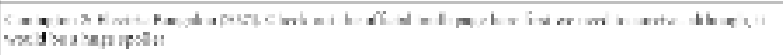 \\
\hline
\end{tabular}

Figure 5 


\section{FINDINGS AND DISCUSSION}

\section{Instagram: Quantitative Analysis - Broad Observations}

After reviewing memes across the four Instagram pages on the days surrounding specified news stories, a total of 307 memes were examined. The collected data displayed a range in the number of regular postings between accounts. For instance, the account "Memes.com" posted the most frequently, with an average of 15 posts per the dates of analysis. In contrast, the account "Daquan" posted less frequently with an average of only 7 posts per the dates of analysis. Although there is a disparity in the number of posts analyzed between accounts, this did not significantly affect the examination of meme engagement with current events. In fact, although "Memes.com" posted the most memes with a total of 103 postings, only $50 \%$ of said memes were related to COVID-19. "Daquan" on the other hand, had a total of 46 postings that were observed, but had $76 \%$ of said memes relating to current events. These findings illustrate that the number of posts within accounts do not hold significance in the data collection process. Each meme account contributes unique critical data that displays an assortment of meme types for further investigation. Additionally, each account provides a concrete foundation to examine regular meme engagement in comparison to memes specific to current events. Measuring this data within and between accounts assists in creating a comprehensive image of the value memes can hold during crises.

Looking at the engagement of memes across all accounts on the specified days of analysis, a total of $13 \%$ of memes were specific to the examined news stories. Although there is no definitive justification for this low percentage, several factors are likely to be causing a low 
correlation. To begin, the month of March marked a large global transition and abundance of new information due to COVID-19. This transition caused an oversaturation of news stories regarding the pandemic including the implementation of new policies, the rise in the number of patients as well as safety precautions. Due to the higher volume of news stories reported during the time period of analysis, the specific major news stories analyzed may not have had a significant impact on the formation of memes as expected. Additionally, the news stories analyzed, although crucial stories in a broad perspective, may not have been significant for the primary demographic meme pages are catering to. For example, a global event that receives a significant amount of media coverage such as the Olympics was not discussed once within the analysis. This may be due to the fact that it is not an issue that is directly relatable to the generation communicating with memes. From these results, a generalization that memes do not have a strong correlation with current events could be interpreted. However, when considering all topics the 307 analyzed memes covered, the data indicates that $69 \%$ of total memes analyzed were related to COVID-19. These results reinforce the idea that the news stories explored may not have played a significant role due to the large number of competing news stories at the time. However, the topic of the pandemic itself played an unmistakable role in communicated memes during the month of March across the examined Instagram pages. The contextual memes related to COVID-19 were used as a framework for a content analysis that will be discussed further. This information will give greater insight on whether particular news stories receive significant attention due to a lack of relatability from its primary demographic.

Of the specific news stories analyzed, the narrative with the most meme coverage was the story about American border closures, contributing to $6 \%$ of memes across all four accounts. 
This is almost half the total percentage of story specific memes observed throughout all data collected. This is a critical observation to mention due to the fact the story itself is one that affects the American youth, whose voices are often omitted from traditional mainstream media sources. The high prevalence of memes surrounding this topic over other news stories examined reinforced the idea that memes are a significant tool utilized to reflect viewpoints and voices of the often overlooked younger generations. These results also showcase an alternative method of voicing a perspective that otherwise would be lost. Other news stories that directly related to younger generations, such as the American school closures, contributed a total of $3 \%$ of all examined memes. This is almost a quarter of all story specific memes observed. It is understandable that there would be a high correlation of meme posting due to the nature of the subject. Once again, the story itself directly impacts the primary generation consuming memes. Although this is the case, there is more attention to the voices of the youth during these stories in both mainstream media, school boards and within peers that the memes become a relatable trend more than a powerful outlet for emerging voices. The higher sense of urgency to share perspectives regarding the American border closures therefore may be the primary reason for its prevalence across all meme accounts observed.

Additionally, it should be noted that all story specific memes revolved around American news stories over Canadian stories. Although closures of borders and schools were measured when this occurred in Canada and the United States, Canadian stories did not evoke the production of any story specific memes from the observed Instagram pages. This may be due to the fact that the pages analyzed originated from the United States and therefore garnered greater interest in American current events. Future research may benefit from the analysis of country 
specific meme pages for more information regarding the production of memes and cultural implications meme pages hold in various nations.

Instagram: Quantitative Analysis - Relationship with Followers

As the activity of four Instagram pages were monitored, observations were made on whether the amount of followers each account had shared a relationship with the number of current event related memes that were posted. These observations were examined through manipulating existing data collected for the quantitative analysis. To access the raw data that was manipulated to observe these findings, see Appendix B.

After the total number of story specific memes were calculated across all given dates of analysis, "Kalesalad" had a significant lead above all other pages with 21 story specific memes. The remaining accounts all had less than 10 story specific memes that were analyzed. A possible explanation for this may be due to the fact that "Kalesalad" has the second highest number of daily meme postings, behind "Memes.com." However, even though "Kalesalad" often tackles story specific issues, the account itself has a relatively low follower count compared to other observed pages. The account has 3.9 million followers whereas the others have almost triple the number of followers. Meme accounts were then explored to find the percentage of COVID-19 related memes outside of the story analyzed to have a better understanding of the relationship between the account's content and its followers. Upon this investigation, "Kalesalad" no longer held the upper hand. Instead, memes from the account "Daquan" had the greatest percentage of 
memes surrounding COVID-19, with a percentage of 76\%. Although Daquan has a lower average frequency of posts, the content of the memes themselves contributed to the account's popularity.

Nonetheless, a direct relationship between the follower count and the content of memes cannot be definitively determined. For example, "Daquan" holds the highest percentage of COVID-19 related memes and has the most followers with 15.5 million. However, the same data identifies "Thefunnyintrovert" to hold the second highest percentage of memes, but has the least number of followers with a total of 2.5 million. Both accounts also post the least frequently, but the memes deemed necessary to share are often related to current events instead of unrelated topics. The stark difference between follower counts within the results indicate that there are a variety of unexplored factors that contribute to the number of followers an account will obtain. These include but are not limited to the age of the account, popularity the account may have on other social media platforms, as well as whether the accounts are buying followers. An analysis of the several factors at play are beyond the scope of this paper, but something that would be beneficial to analyze further in future research. For the remainder of this paper, greater emphasis on the metrics of likes and comments will be interpreted to understand engagement and the popularity of memes.

\section{Instagram: Quantitative Analysis - Understanding Likes and Comments}

In order to understand the level of engagement memes discussing COVID-19 receive, a comparison of the number of likes and comments surrounding memes relating to COVID-19 and other general memes were conducted. Here, 'general memes' are defined as all memes posted that are not specific to COVID-19. From this comparison, similar patterns were observed across 
all four Instagram pages, but will be exemplified in depth using the account "Memes.com". This account was chosen to illustrate the general patterns identified because the account holds the largest number of total postings. Furthermore, "Memes.com" also has the widest range of meme types. In fact, only $63 \%$ of memes observed from "Memes.com" were related to COVID-19. The diverse meme types allow for a close even comparison of metrics between general and COVID-19 specific memes. Figures 6 and 7 illustrates the level of engagement in the forms of likes between the two categories.

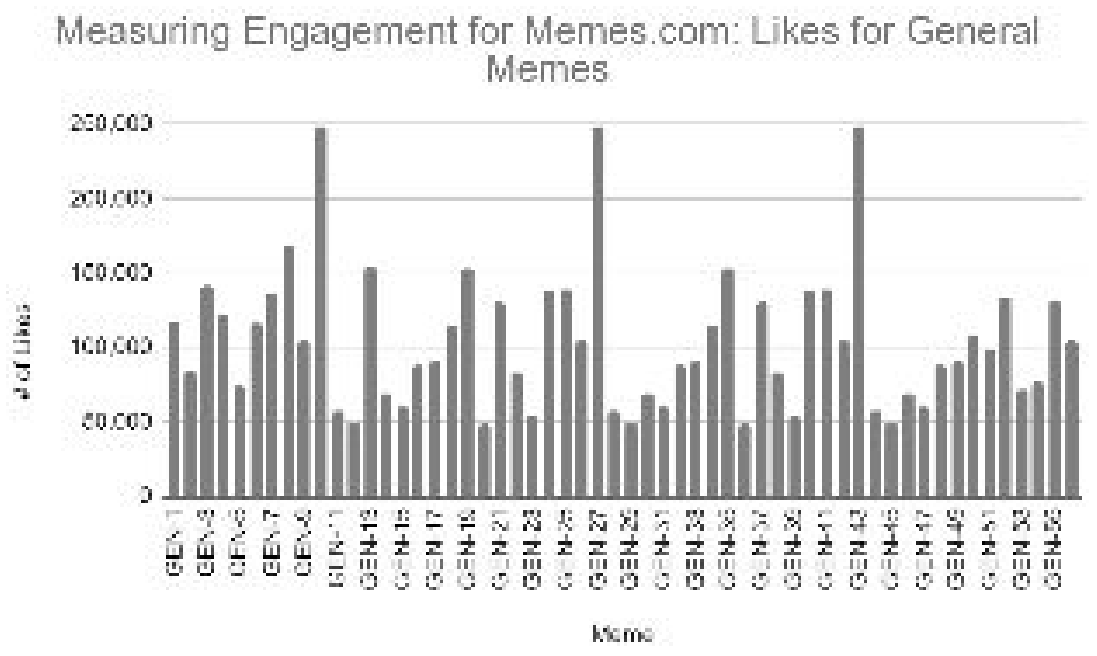

Figure 6 


\section{Measuring Engagement for Memes.com: Likes for COVID-19 \\ Specific Memes}

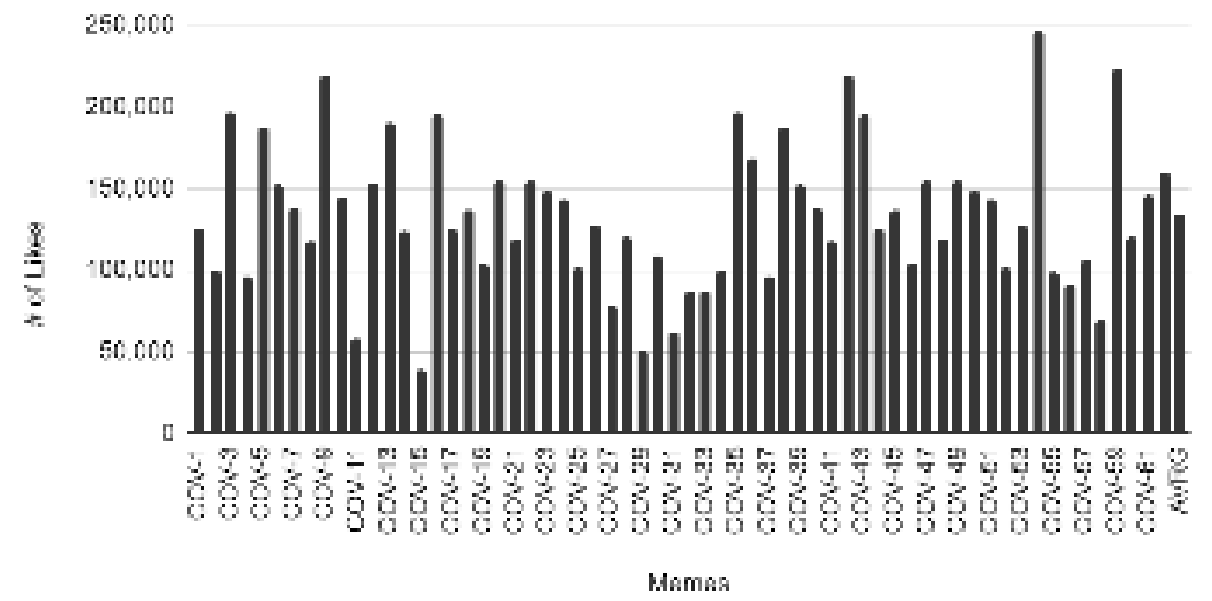

Figure 7

Evidence from Figures 6 and 7 showcase that there are a significantly higher number of likes accounted for memes discussing COVID-19 in comparison to general memes within the same timeframe. More specifically, the average number of likes recorded for general memes is 104,703 likes, whereas the average number of likes for memes related to COVID-19 is 133,827 . These findings reinforce that within the same timeframe, audiences are choosing to engage further with COVID-19 related memes than others. The patterns of engagement also demonstrate that followers are seeking to interact with a particular type of meme over others. At the height of confusion, individuals are seeking memes that feel relevant, relatable and keeping a younger generation in mind. This is critical for the primary meme demographic.

The prevalence of mood management may also be a crucial factor within these results. Studies indicate that when audiences are engrossed within a general mood or emotion, the online content they seek out subconsciously matches this emotion (Kien, 2019). As individuals connect 
with content surrounding COVID-19, they are "motivated to choose information that follows from what they have last consumed to maintain and possibly add to the feeling" (Kien, 2019, p.54). The partially subconscious emotional connection to memes related to COVID-19 encourages further engagement that may be lacking in more traditional media outlets. Similarly, studies conducted by Weng et. al (2012) acknowledge the high number of memes posted daily and identify the common factors necessary to measure the virality of one meme over others. Their studies state that "the combination of social network structure and competition for finite user attention is a sufficient condition for the emergence of broad diversity in meme popularity" (Weng et. al, 2012, p.6). However, their findings suggest a strong relationship between a meme's longevity of virality and current events. Further analysis indicated that "long-lived memes might be sustained exogenously by traditional media and real-world events" (Weng et. al, 2012, p.4). These observations once again highlight the motivation for higher engagement with memes surrounding the pandemic. As mainstream media and public anxieties keep COVID-19 relevant, related memes also encourage relevance. High engagement with these memes enables a rich outlet for communication and connection.

Previous findings are reinforced further when analyzing the consistency of high engagement shown in Figure 7 in contrast to the inconsistent spikes of engagement seen in Figure 6. Figure 6 illustrates that some general memes do receive just as many likes, if not more than memes surrounding COVID-19, but struggle to obtain and maintain this type of attention. A general meme needs to be exceptionally funny or engaging to receive the same type of engagement that a meme related to COVID-19 would be able to easily obtain. However, when 
analyzing meme engagement in the form of comments, similar results are not found. This data is displayed in Figures 8 and 9.

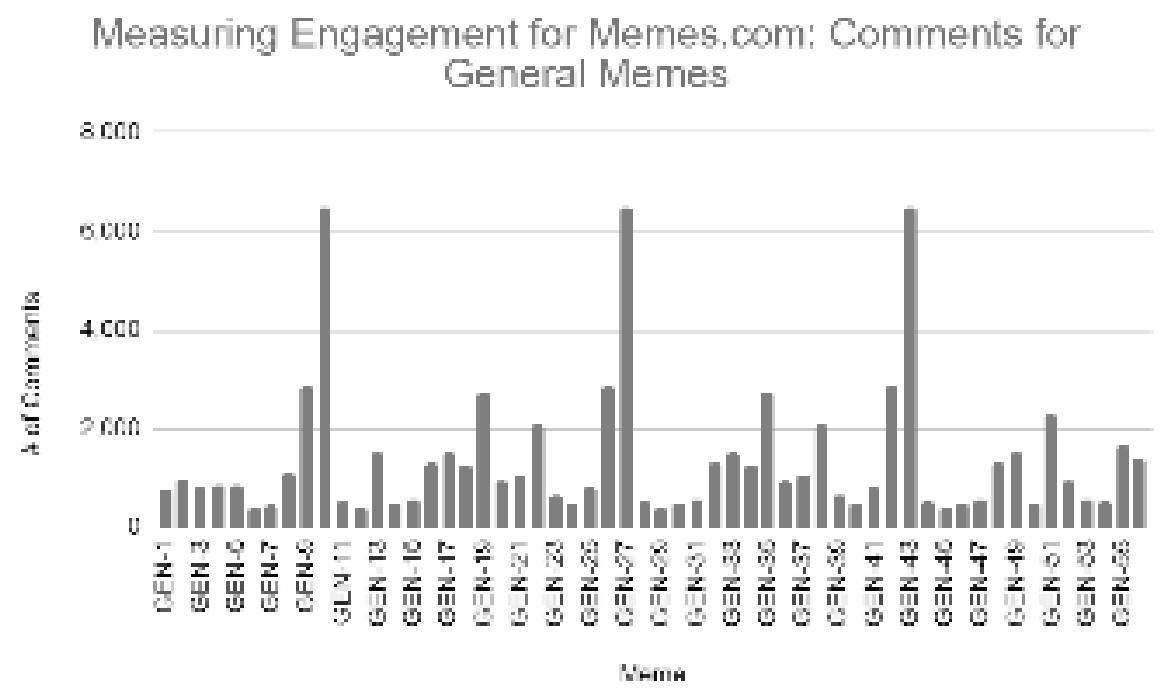

Figure 8

\section{Measuring Engagement for Memes.com: Comments for COVID-19 Specific Memes}

$0,0 \mathrm{x}$

8.000

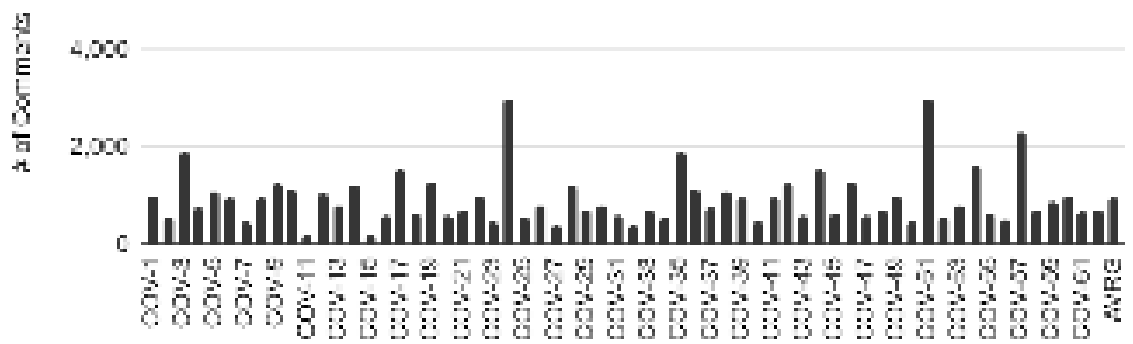

Swmen

Figure 9

Figures 8 and 9 uncover that there is a greater number of comments surrounding general memes than there are of COVID-19 specific memes. The overall data respectively shows that there is an average of 1,401 comments for the general memes category, whereas the average for 
COVID-19 specific memes is 952. Unlike data comparing the number of likes between categories, there is a greater number of inconsistencies between the number of comments each meme received. General memes did receive higher spikes in the number of comments a post received, but was only the case for 3 specific memes out of the 55 examined. This data illustrates that memes have a difficult time enabling new discourses surrounding COVID-19 than other relatable general memes. The result of this may be due to several factors including discomfort on the subject, a lack of knowledge surrounding the topic or being uncomfortable engaging on the particular platform. It is also important to note that the same memes that gained a high average of likes, 133, 827 likes, are receiving a very low average of comments, 952 comments. This shows that the data is not only displaying a lack of engagement through the form of comments, but a lack of interaction, a key affordance Instagram presents to its audience.

The disparity between audience engagement in the form of likes and comments may not have to do with the memes themselves, but can also be due to the platform's practices. As Instagram is primarily a photo sharing platform, the lack of comments may be due to the unique affordances Instagram brings in comparison to other social media platforms. Platforms such as Twitter are primarily text based, and may be where further discourses can be uncovered. Instagram then, can be seen as taking an active role in consolidating thoughts and comments surrounding youth and current events that can then fuel further discussion and activism on other channels. This idea is reinforced when noting the emergence of screenshots of Tweets posted as a meme across all Instagram pages analyzed. Here, Instagram acts as a medium to amplify voices that advocate for youth engagement and provide the opportunity to be continued on another platform entirely. Although this paper will delve into the interplay between discourses within 
Twitter and Instagram, I encourage future studies to further examine the comment sections of meme accounts on Instagram to obtain a better understanding of commenting practices taking place.

Instagram - Emerging Trends: Tweets as Memes

To better understand the types of content receiving the most engagement, the most liked and commented memes surrounding COVID-19 from each account were analyzed. Memes were selected and compiled into a sample of 29 images, excluding all repeating memes. One of the most prominent observations encountered from the analysis of this sample was that $59 \%$ of memes were reshared images of popular Tweets on Instagram. These images are redefining what is accepted as a meme, as traditionally a meme would consist of a remixing of recognizable images with often socially charged text. The resharing of Tweets from Twitter, however, removes the aspect of imagery within a meme altogether by forcing the larger discourses of the Tweet to be at the center of attention. Rather than traditional internet memes, this form of meme is often referred to as "emergent memes". These memes are "spreadable media" that are not "iterated and remixed further as separate contributions", but instead, reposted as an internet meme on Instagram (Bowers and Wiggins, 2015, p.12). The prevalent shift of what is presented and understood to be a meme on Instagram is one that has yet to be explored by scholars. For the remainder of this paper, emergent memes of this manner will be categorized as a traditional internet meme. The frequency of occurrences on the observed Instagram pages indicates that this form of meme has already been widely accepted across its audiences and continues to receive positive engagement. My findings will attempt to break down the significance of this shift, but I 
encourage future research on the implications of the relationship between the two prominent social media platforms.

The emergence of Tweets on Instagram's meme pages, especially as memes with the highest number of likes and comments, show that audiences desire to interact with raw and informative content. Platforms such as Twitter and Instagram provide a unique opportunity to discuss the mundane that is often overlooked by traditional media outlets. These forms of memes encourage greater critical thinking and offer an opportunity to discuss new elements of a current issue. Small scale discussions then have an environment to proliferate into more meaningful discussions about larger events. Often, the sarcastic or entertaining tone to many of these Tweets are what allows it to be successful in the meme format and create further engagement. Crafting tweets in this manner becomes a communicative skill similar to the constructing of traditionally observed memes. This is exemplified in Figure 10, a tweet posted on one of Instagram's meme pages. The tweet's satirical tone towards a relatable topic concerning COVID-19 presents a communicative vessel that is able to create meaning from the mundane. Accepting this form of tweet as a meme indicates a meme account's desire to broadcast more than entertainment to its audience. Memes can be seen as being selected to translate current events through unique perspectives.

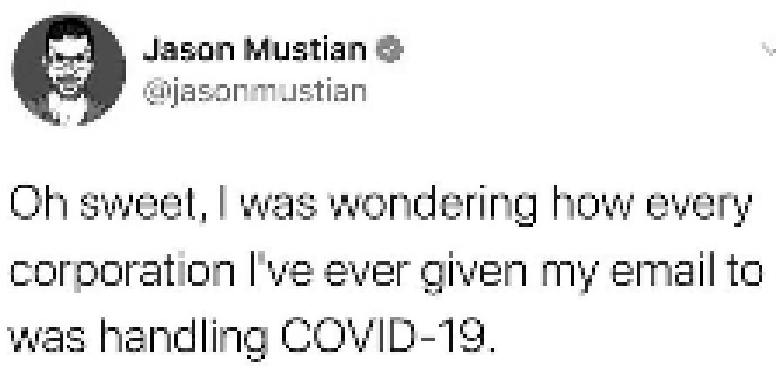


Figure 10

Twitter is also a space where a variety of users reside. This includes corporations, large news outlets and celebrities. The wide array of audiences mixed with a user's personalized algorithm makes consuming content from regular users more difficult. Instagram magnifying the voices of users who are 'unverified', determined by the lack of blue checkmark by the name as seen in Figure 10, encourages three leading objectives. Firstly, meme pages are encouraging its audience to acknowledge that memes hold more power than just as a mode of entertainment. The page's choice to strip away videos and photos to go alongside text in a number of posts urges its audience to acknowledge the relatable consequences young generations are currently facing. Secondly, sharing Tweets on another platform creates a stronger sense of community. The Instagram page is able to act as a megaphone for its primary demographic. Audiences observing the images of Tweets displayed are able to take comfort in the fact that their voices are being heard and appreciated by others within their age group that may otherwise have gone unheard. Recognizing the relationship the two platforms have may also encourage users who are not already on Twitter to create an account to share similar or opposing thoughts as well, now that they are aware that others share similar values. Finally, sharing Tweets on Instagram facilitates further discourse. Meme posts in the form of Tweets give its audience a simple mechanism to discuss and critique complex issues to an age group often dismissed from discussions. Even if a tweet only covers small instances, starting the conversation itself will ripple into further discourse about large-scale events. Examples of these types of memes can be found in Figures 11 and 12 . 


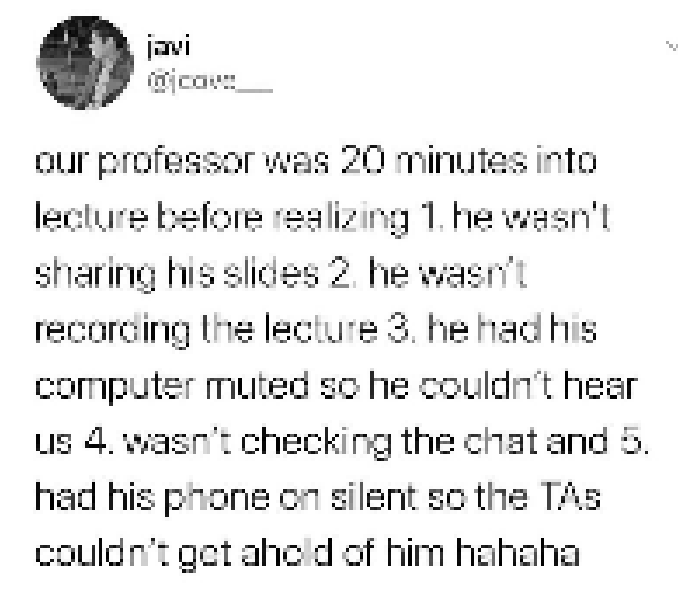

Figure 11

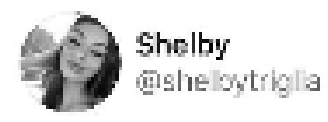

Damn my boyfriends job really said for the next 2 weeks if they dont want to work they will get full pay but if they want to work they will make double time... NOW THATS HOW YOU TREAT YOUR ENPLOYEE'S!

Both figures 11 and 12 share mundane experiences that seem relevant and relatable, while also serving as a tool to facilitate larger discussions about the veiled social issues within these instances. For example, Figure 11 shares a humorous anecdote about a class, but aims to facilitate larger conversations surrounding COVID-19 and the consequences online learning has on its students. Similarly, figure 12 shares another personal anecdote revolving around the topic of work experience during the pandemic. This meme also encourages discussions about the broader treatment of essential workers during unprecedented circumstances. Instagram displaying personal anecdotes in this manner acts as a repository of the common themes and anxieties an entire generation, as well as society, experiences first hand. Once audiences observe these memes, even if they decide not to engage in further discourse, they obtain greater insight on the consequences of COVID-19. This information allows the platform's audience to make greater informed decisions based off of the distributed information meme accounts provide. 
The rise of Tweets on Instagram also assists in responding to one of Grant Kien's leading critiques in his work Media Memes and Prosumerist Ethics: Notes Toward a Theoretical Examination of Memetic Audience Behavior. Kien states that one of the behaviours audiences consuming memes will encounter is "armchair activism” (Kien, 2013). He characterizes armchair activism as "the extent of many people's political involvement”, since meme consumers believe "they are doing something to change the world by simply forwarding information to their already existing electronic friendship network without any depth of dialogue about the topic" (Kien, 2013, p.557). The interplay between Twitter and Instagram observed attempts to break this limitation through amplifying voices through the formation of memes from Tweets. This encourages its audiences to vocalize their own opinions over only participating in the practice of sharing. In this case however, the practice of sharing allows for voices to be amplified. The dual purpose of Instagram's meme pages is that it creates a space that cultivates dialogue of critical discourse while simultaneously spreading awareness.

\section{Instagram: Content Analysis}

After conducting a content analysis of the most liked and commented memes observed, prevalent underlying themes were identified. The analysis determined that the most liked and commented memes exhibited almost identical results with a difference less than $5 \%$ between categories. Taking this into consideration alongside the fact that Instagram showed higher engagement of likes than comments, my findings will delve further into the examination of the most liked memes. Figure 13 illustrates the prominence of identified themes within a sample of 18 memes. This data will assist in uncovering the relationship memes share with mainstream 
media, while also exploring how memes provide a channel for understanding current events that is relatable and engaging for youth consuming this form of communication. From this display of data, there are three critical themes that emerge over all other nuances explored.

\section{Most Liked Memes: Breakdown of Common Themes}

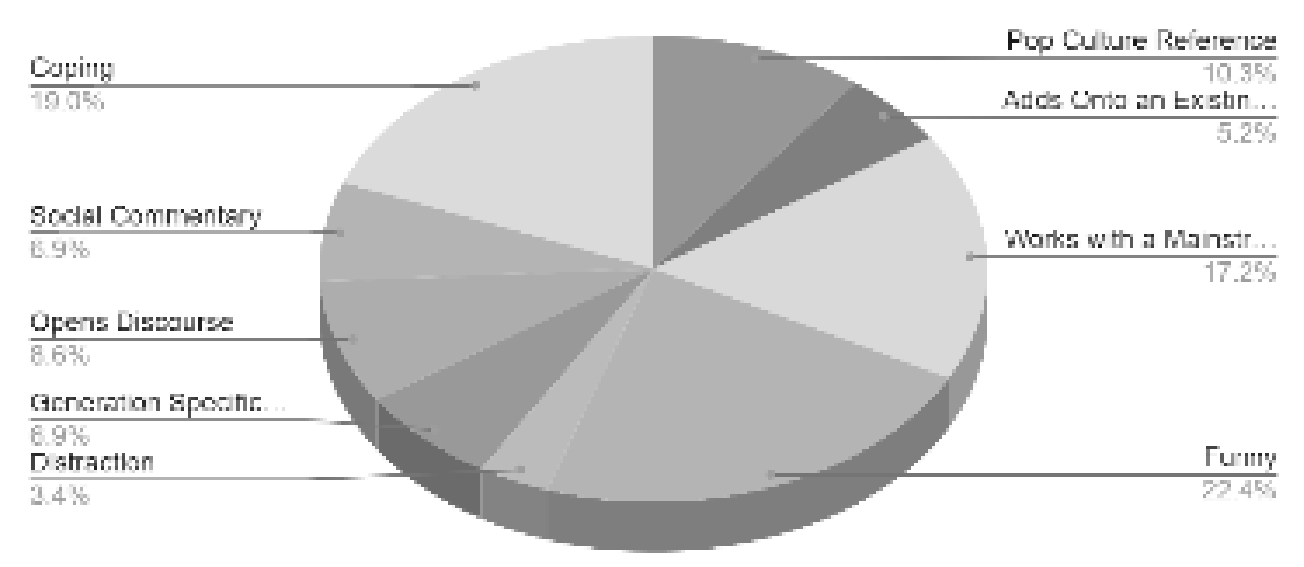

Figure 13

Figure 13 indicates that the terms "funny", "works with mainstream media" and "coping" are the three most prominent themes that emerged from this analysis. The prevalence of memes categorized as "funny" is one that is expected, since memes are primarily characterized as a funny and entertaining mode of communication. Although this is an expected dominant theme, it is noteworthy that this theme encompasses $22.4 \%$ of the sample. This shows that although "funny" is a dominant theme, it sums up less than a quarter of all memes analyzed. The lack of a greater presence indicates that more refined nuances behind memes are often overlooked. For example, memes that dealt with coping or acted as a coping mechanism occurred almost as frequently as memes fell under the category "funny". In fact, $19 \%$ of the sample fell under the 
"coping" category. Similarly, memes that worked with mainstream media occurred in $17.2 \%$ of the sample. These themes making up a large portion of the content analysis among categories such as "funny" demonstrates the reliance users have on memes to provide information and comfort about what dominant media sources are currently reporting on. The fact that these categories are emerging within memes with the highest engagement also implies the audience's expectation for meme pages to provide commentary on relevant real world events that users are able to interact with.

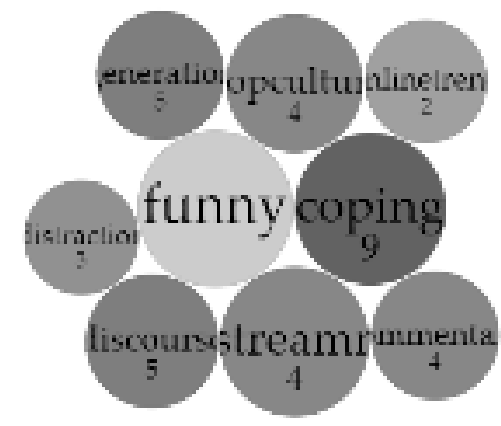

Figure 14

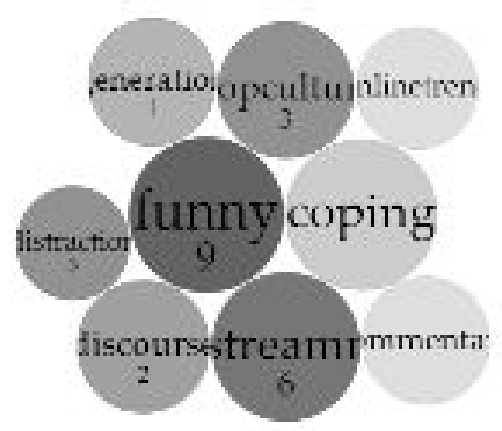

Figure 15

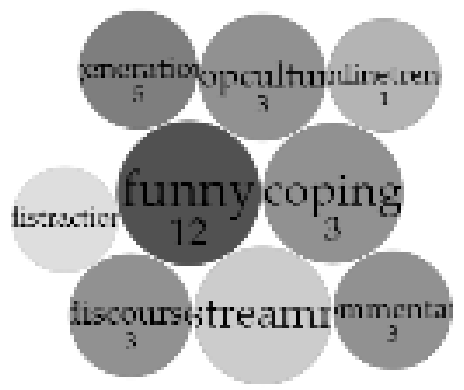

Figure 16

Figures 14, 15 and 16 illustrate the relationship between the discussed leading themes. Each theme highlighted in dark grey is the theme of focus, while pink bubbles and numbers surrounding each are the number of times these themes occurred together. The darker shade of grey also represents the level of dependence between relationships. A considerable amount of the 
sample displays that memes with high engagement attempt to utilize all three themes to capture the attention of its audience. From the visualized data, the strongest relationship that can be observed is between "works with mainstream media" and "funny". Of the sample size observed, $67 \%$ of memes fell between these categories. The observation between themes displays a dependent relationship successful memes have with current events and mainstream media. This shows that Instagram's meme pages are choosing to display popular memes that take trending stories to create humour, rather than displaying other unrelated visuals that coexist within the meme-verse.

Accentuating these findings is the work of Olivia Rose Marcus and Merrill Singer in Loving Ebola-chan: Internet Memes in an Epidemic. The usage of imagery of their identified "Ebola-chan"uncovers that the presence of the viral character goes beyond a comedic relief and coping mechanism (Marcus and Singer, 2017). Instead, the internet "trolls" were seen intentionally spreading rumours surrounding the virus and utilizing imagery of Ebola-chan to comment on the "way the media spreads images and messages of fear, disaster, and terror on a daily basis through a more accessible and socially approved medium" (Marcus and Singer, 2017, p.349). The spread of false information for comedic purposes and humorous imagery surrounding the virus, served as "a form of violence or rebellion against mainstream hype over an unspeakable tragedy" (Marcus and Singer, 2017, p.351). As COVID-19 falls within a similar category, comparable findings and forms of rebellion are expected. In fact, a Corona-chan image has already surfaced within the realm of memes. As Marcus and Singer indicate, the rise of memes regarding a large epidemic and the rise of alternate imagery rebel against the cyclical and sensationalized imagery produced within mainstream media. This rebellion can be seen within 
the content analysis, which reinforces the notion that memes are amplifying new voices, often from an underrepresented demographic: society's youth.

Similarly, the prominence of "coping" coexisting within these themes also highlights that memes are attempting to provide a medium to understand and reflect on current events at a pace that mainstream media sources are unable to. Memes comment on mainstream media, but also comment on the emotional toll felt by its young audience. Whereas mainstream media may cause "the spread of moral panics and mass hysteria" due to the "ideas spread through media" (Kien, 2019, p.78), memes can be seen breaking down panic by creating emotionally manageable content. "Coping" memes are able to capture the anxieties its audiences are expressing through Instagram comments as well as its established relationship with Twitter. These platforms are then able to amplify memes that speak to the anxieties this same audience is feeling. Marcus and Singer (2017) also identify that mainstream media tends to focus more on the sensationalization of stories over the emotional toll that may burden its audience. When mental health is discussed, experts are often interviewed to provide advice and reassurance to their viewers. However, several of the discussions taking place considering coping strategies are often not targeted towards younger demographics.

Lacking representation and guidance in this manner, memes attempt to bridge the gap through addressing these events with humour and targeted relatability. For instance, Figures 17 and 18 display memes that were coded to fall under the categories "coping", "funny" and "works with mainstream media". A closer look at these memes reinforces how the three themes work 
together to fulfill a sense of togetherness within the meme community as they attempt to depict common anxieties in a light hearted manner.
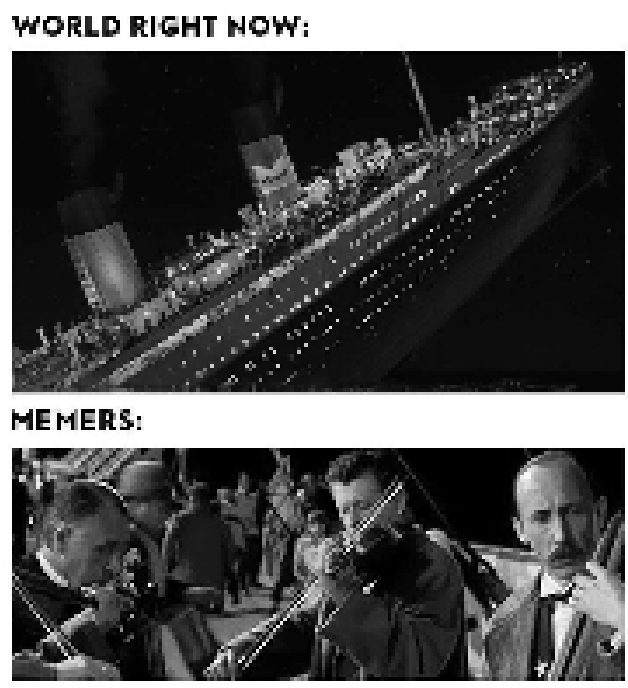

Figure 17

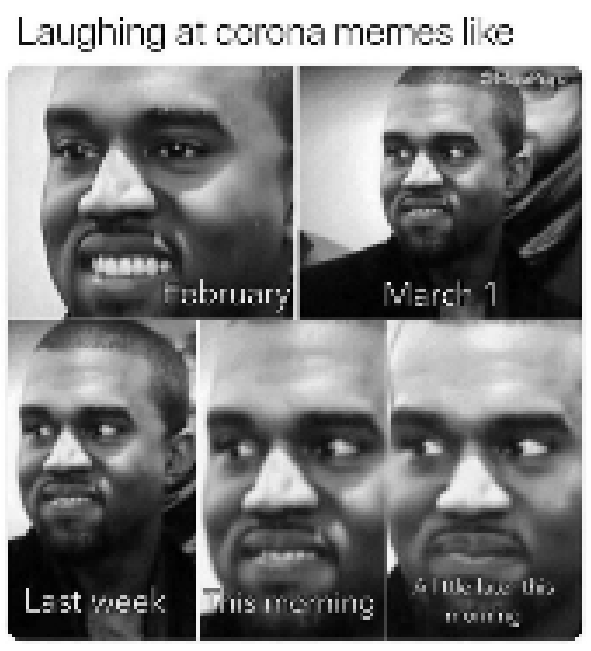

Figure 18

Figure 17 specifically utilizes a pop culture reference to illustrate how "memers" are coping with their given circumstances surrounding COVID-19. Here, the meme quite literally displays the message of pushing through and finding sources of entertainment amidst a disaster. The meme also assists in displaying a method of coping while simultaneously providing a coping mechanism through the usage of comedy surrounding current events. Similar utilizations of current events, comedy and coping mechanisms can be examined Figure 18. This meme also uses a pop culture reference, the next most coded theme discovered, through using a prominent figure, Kanye West. Kanye's increased expressions of concern mimics the concern young demographics are likely feeling upon the progression of current events surrounding COVID-19. The analysis of these two memes utilizing the prominent themes displayed within the content analysis demonstrates how memes are responding to current events and its necessity to do so. 
Although the key demographic observing these memes may not be represented in other forms of media, having a "safe space" to interact with their feelings of concern is sought out and appreciated, determined by the high engagement observed.

It is also important to note that Figure 13 displays a low percentage of memes corresponding with the themes "social commentary", "opens discourse", "distraction" and "generation specific". The cause for the low correspondence of memes surrounding social commentary and further discourse likely coincides with the discovered emergence of tweets as memes. Rather than memes found on the platform insighting discourse itself, memes amplify discourses occurring on Twitter, encouraging users to take part in similar activities on another social media based platform that revolves around sharing opinions. Additionally, the posting of COVID-19 specific memes itself is already rebelling against mainstream societal norms through the rise of new voices and commentary, as mentioned by Marcus and Singer (2017).

An example of one of the most liked memes that does fall under both the "social commentary" and "opens discourse" themes can be seen in Figure 19. This meme perfectly illustrates the interplay between Twitter and Instagram. The amplification of Tweets as a meme format such as this is reinforced when considering that the meme received a total of 93,458 likes, 41,681 more than the average number of likes within the data set. The meme attempts to poke fun at the fact that before you can isolate yourself responsibly, individuals must put themselves into danger to get needed supplies. 


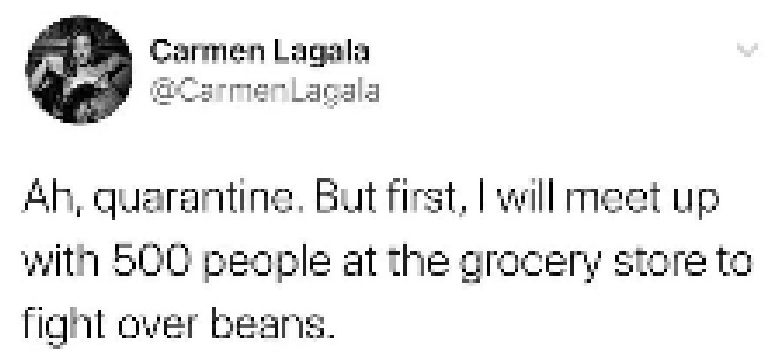

Figure 19

The themes "distraction" and "generation specific memes" are also observed to be low within the content analysis conducted. It is interesting to note that the "distraction" theme is not prevalent within the sample. This theme only occurred within $3.4 \%$ of the content analysis, the lowest percentage between all themes analyzed. The lack of engagement with the theme "distraction" emphasizes the audience's desire to seek memes as a method of information over entertainment. Although both are present within a meme page itself, information regarding current events, whether this is displayed anxieties, remarks or news stories, takes precedence over a desire to consume memes purely for entertainment. Additionally, "generation specific memes" did not prevail as frequently as anticipated. Figures 20 and 21 display examples of generation specific memes that were uncovered. These memes are displayed emphasizing their generation by opposing others by referencing "boomers" and making references to generationally familiar objects such as childhood games. Although memes such as these do exist, the majority of memes analyzed do not attempt to exclude generations from partaking in discourses memes bring forth. Rather, memes are working to include unheard voices in a manner that appeals to particular generations without excluding others who may want to participate. As Grundling (2018) notes, memes can be observed as a communicative speech act. This means that 
"if an addressee is not able to recognise the speech act, successful communication is not possible, since recognising the speech act provides the addressee with appropriate constraints for his/her response(s)" (Grundling, 2018, p.152). Observing memes as speech acts therefore attracts younger generations with a larger understanding of the communicative practice, but does not exclude other individuals outside of the younger generation to utilize this form of communication. This displays the function of a meme as a tool to integrate new conversations within current events in a method that is comfortable, inclusive and disruptive.

Me: I guess I could handle 4 weeks of quarantine

\section{Government:}

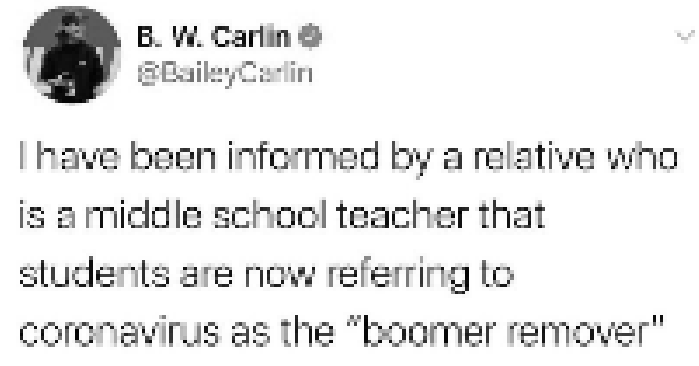

Figure 20

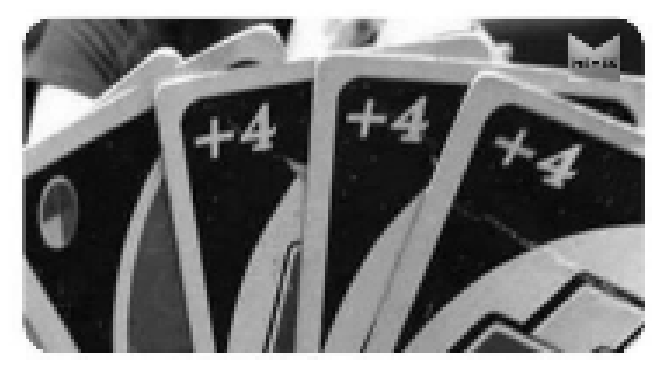

Figure 21

\section{Reddit- Overview of Results}

Upon conducting a content analysis of the top five memes identified within the subreddit $\mathrm{r} /$ coronavirusmemes and performing a critical discourse analysis on the associated comments, it was identified that overall, the platform did not produce any productive discourse. This outcome may be due to several factors surrounding the subreddit itself. One major factor to explain this phenomenon is that the average age demographics for Reddit users is higher than Instagram 
users. The age range of Reddit users varies and is more evenly split than the age range found on Instagram. For instance, on Reddit, " 22 percent of internet users aged 18 to 29 years and 14 percent of users aged 30 to 49 years" (Sattelberg, 2020, pp.7) whereas on Instagram, 30\% of their global users are the ages of $18-24$ (Omnicore, 2020). Similar data also shows that $72 \%$ of US teens use Instagram (Anderson and Jiang, 2020). A higher age range within Reddit coincides with greater representation within mainstream media, meaning the motivations behind the utilizations of memes may stray away from the emergence of unheard voices and move closer to acting as a form of entertainment.

Another distinct factor that may contribute to the lack of productive discourse may be due to the environment examined. Instagram as a platform is primarily a photo sharing app where memes are able to thrive by incorporating current events with the primary medium Instagram promotes. In contrast, Reddit allows its users to join more specific and meaningful subreddits that are able to engage with current events of their choosing head on. Since information and meaningful discourse is more readily available within designated subreddits, the utilization of memes within the platform focuses less on creating discourse and more on the shock and entertainment value they hold. Additionally, the subreddit allows anyone within the community to post memes, whereas Instagram pages are regulated by a much smaller subset of individuals. As Reddit emphasizes community collaboration, stronger messaging through memes could potentially be lost. However, analyzing the level of engagement through upvotes identifies that despite a larger quantity of memes emerging from multiple users, the memes with the highest upvotes generally do not encourage critical commentary or discourse. This suggests that users 
are choosing to engage with the subreddit in a more light hearted manner than Instagram's primary audience.

Finally, Reddit as a platform often encourages its users to comment with funny and sarcastic remarks, in an attempt to receive upvotes from other users based on how entertaining or informative others perceive their comment. They may also receive "Reddit Gold" that once again entices users to cultivate such responses in order to be rewarded with higher engagement. Based on these additional motivations, the quality of the overall discussion diminishes in subreddits that do not establish strict guidelines on the type of discussions that can occur. As Kien (2013) notes, social media platforms such as Reddit, where participation of users shape the user's experience risk falling into the "hive mind" phenomenon (Kien, 2013). Here, the subreddit's community falls at risk of becoming "solipsistic and self-referential" and "preferring a mentality of groupthink" that may not engage with discourse surrounding "challenges or influences that might under normal circumstances improve the strength of their community and new intellectual breakthroughs" (Kien, 2013, p.556). Similarly, Literat and van den Berg (2019) observe that "subcultures, such as those within Reddit, often engage in subversive play as they establish their community membership against a dominant discourse or mainstream norm" (Literat and van den Berg, 2019). This reinforces Reddit's discourses strive to intentionally shock and play against mainstream media news stories over the significance of critical discourse. Due to these outcomes, only a broad overview of findings will be illustrated as the content analysis does not prove to contribute to the larger nuances this paper attempts to uncover. 


\section{when I am watching "Covid-2019" movie in ten years, I hope I will see Matt Damon panic buying toilet papers.}

Figure 22

Figure 22 displays the most upvoted meme on the subreddit $r$ coronavirusmemes. The meme primarily utilizes a pop culture reference and humour to captivate its audience. Although the meme discusses panic buying toilet paper, the overall context moves away from discussing the social ramifications of this issue and instead focuses more on the current and future entertainment the situation will bring forth. Referring to Matt Damon's previous movie regarding a fictional pandemic entitled "Contagion", the meme focuses on fantasizing how a sequel would emerge. Figure 22 also illustrates that the most commented meme on the subreddit is an image of a tweet taken from Twitter, similar to findings identified on Instagram. However, the productive discourse surrounding the meme itself and subsequent comments demonstrates a lower critical engagement with current events. Figure 23 breaks down the most prominent themes that surface through a content analysis of the comments associated from the post. 


\begin{tabular}{l|r|r|}
\multicolumn{1}{c|}{ Post 1 Major Themes } & \# of Occurances & Percentage \\
\hline Theme 1: Pop Culture Reference & 15 & $26.79 \%$ \\
\hline Theme 2: Political/Social Commentary & 8 & $14.29 \%$ \\
\hline Theme 3: Funny & 18 & $32.14 \%$ \\
\hline Theme 4: Off Topic & 8 & $14.29 \%$ \\
\hline Theme 5: Asking a Question & 3 & $5.36 \%$ \\
\hline Theme 6: Coping & 4 & $7.14 \%$ \\
\hline Total & 56 & $100.00 \%$ \\
\hline
\end{tabular}

Figure 23

The breakdown of data displayed above indicates that the majority of the comments examined fell under the "funny" or "pop culture reference" category. It is also crucial to note that there is an equal amount of social and political commentary as there is off topic commentary. This suggests that the focus of discourse the platform promotes moves away from critical social analysis and more towards a coping mechanism through humour and distraction. A critical discourse analysis of the comments under the meme displayed in Figure 22 exhibited a stronger focus on conversations dealing with small details the meme utilizes for humour rather than discussions surrounding societal implications the pandemic may hold. Here, Kien (2013)'s fear that "intelligence may be displaced by cleverness" can be observed occurring in full effect ( $\mathrm{p}$. 556). For instance, several of the comments concentrated on the potential title of the movie discussed in the meme, as well as who would be its lead actors and directors. Users commented "Contagion 2: Electric Boogaloo (2021). Check out the official imdb page here first we need to survive. although, it would be a huge spoiler" (logray71, 2020) and "SARS-2 :The Sickening" (Zapdostresquatro, 2020) to contribute to the ideology that users should be looking forward to the movie that will emerge from the pandemic. Numerous comments responded to one another 
such as "I want the Dwayne The Rock Johnson version.” (Joelcamposlapuente, 2020), and "Jason statham and Liam neeson version" (BLKush22, 2020). Although some comments did make references to relevant current events such as "Contagion 2 is filming right now! Starring Tom Hanks!” (KiiWii2029, 2020), referring to Tom Hanks announcing that he was undergoing treatment for COVID-19, the general theme of discourse surrounded hypotheticals that did not contribute to larger discussions about COVID-19.

Overall, the subreddit analyzed from the platform Reddit did not engage in critical discourse as observed with Instagram and its relationship with Twitter. Factors discussed including the disparity in the age ranges of both platforms, the purpose of each platform as well as its interplay with other social media accounts resulted in Reddit's memes playing a less significant role in creating meaningful discussions that heightens and disputes mainstream media news stories. However, the observations examined on Reddit makes an evident distinction of the role memes play. Examining memes from both Instagram and Reddit shows that the suppression or lack of representation of current events for particular age groups and platforms enhance the critical role memes play. The quality of memes and its engagement can be observed increasing as discourses surrounding demographics and current events are limited in online social spaces.

\section{CONCLUSION}

Overall, my research aimed to evaluate whether memes can be utilized to create a socially charged platform that amplifies new and emerging voices. In the midst of a crisis, examining memes as a critical communication tool provided insight into how public anxieties 
were represented and dealt with. The findings within this paper provide definitive answers to the three primary research questions posed at the beginning of this paper.

What is the relationship between memes and mainstream media?

A content analysis of the most popular memes on Instagram and the conducted quantitative analysis determines that successful memes are highly dependent on topics surrounding mainstream media. An overall greater average number of likes surrounding COVID-19 memes across all accounts analyzed suggests that meme audiences are looking to engage with content that addresses socially charged topics. The content analysis also reinforced these observations by noting that an overwhelming majority of the memes collected referred to COVID-19. Finally, the fact that an entirely new subreddit emerged at the beginning of the pandemic supports findings of the relationship between memes and mainstream media. The level of engagement and discourses surrounding memes exhibit a strong dependent relationship between memes and mainstream media.

How do memes provide a channel for understanding current events in the face of more dominant and mainstream discourses?

Analyzing memes receiving high engagement throughout the period of a month showcased that memes contributed to existing conversations surrounding current events with unique perspectives. Many of the memes displayed personal anecdotes that were relatable to a younger generation, while simultaneously encouraging critical discourse on larger social circumstances. Additionally, findings determined a strong correlation between memes receiving 
high engagement and "working with mainstream media" as a category, often associated with humour and coping strategies. From these findings, we can see how memes are attempting to break down larger issues in a manner that is comfortable, informative and more inclusive for a demographic often left out of traditional media. It was also observed that the news stories with the highest engagement associated with memes were those that affected younger demographics whose voices were unheard. Combining these findings together determined that memes work to provide accessible tools for voicing the unheard opinion of the youth.

Do memes empower younger demographics to critically engage with current events?

All findings within this paper point to the fact that memes can, and continue to, play a significant role in providing a comfortable communicative device for younger generations to critically engage with current events. Findings from Reddit reinforce how memes as a communicative device can be used as nothing more than a tool to produce comedy. However, observing platforms consisting of primarily younger demographics shows that this same communicative tool can be used to create profound impact. After determining the strong impact memes increasingly play within our society, using this as a tool to understand a generation can assist in ensuring that emerging voices do not remain unheard.

As current findings indicate that memes provide a window into the voice of the youth, taking the concerns presented here into account will ensure well rounded solutions during a crisis. My findings display that behind the quick snippets of humour memes offer, there are often socially charged statements that seek to reach wider audiences. Recognizing memes as a legitimate communication tool can help lead to further inclusive changes to policies and societal 
behaviours. Upon the completion of this paper, the world is still anxious about COVID-19 and how nations will come together to create a strengthened post COVID-19 world. My hope is that memes will play a larger role in disseminating information that is not easily dismissed, and that they will work hand in hand with the information shared within mainstream media to bring about a better world. 


\begin{abstract}
APPENDIX A
Two online sources were referenced to determine which significant news stories would be analyzed within this study. These sources provided brief summaries of significant events and were then cross referenced with other online news stories and memes. As this paper focuses on North American news stories, a prominent Canadian news source as well as a seperate American News source were explored. References and direct links to these resources will be provided below:
\end{abstract}

The Canadian Press. (2020, April 11). What's happened in Canada since WHO declared COVID-19 a pandemic $\mid$ CBC News. Retrieved from https://www.cbc.ca/news/politics/covid-19-timeline-canada-who-pandemic-1.5529920

Muccari, R., Chow, D., \& Murphy, J. (2020, July 08). Coronavirus timeline: Tracking the critical moments of COVID-19. Retrieved from https://www.nbcnews.com/health/health-news/coronavirus-timeline-tracking-critical-mo ments-covid-19-n1154341 


\section{APPENDIX B}

Disclaimer: All templates, data collection and analyses displayed within this section were solely conducted by myself, Laavanya Srichandramohan, for the purpose of this research paper.

\section{QUANTITATIVE ANALYSIS}

In the quantitative analysis conducted for observed Instagram memes, data was collected as illustrated in the Figure 1 of this paper (and below for reference). For each meme observed within the set parameters of the data collection process, posts were numbered and recorded with its corresponding number of likes and comments.

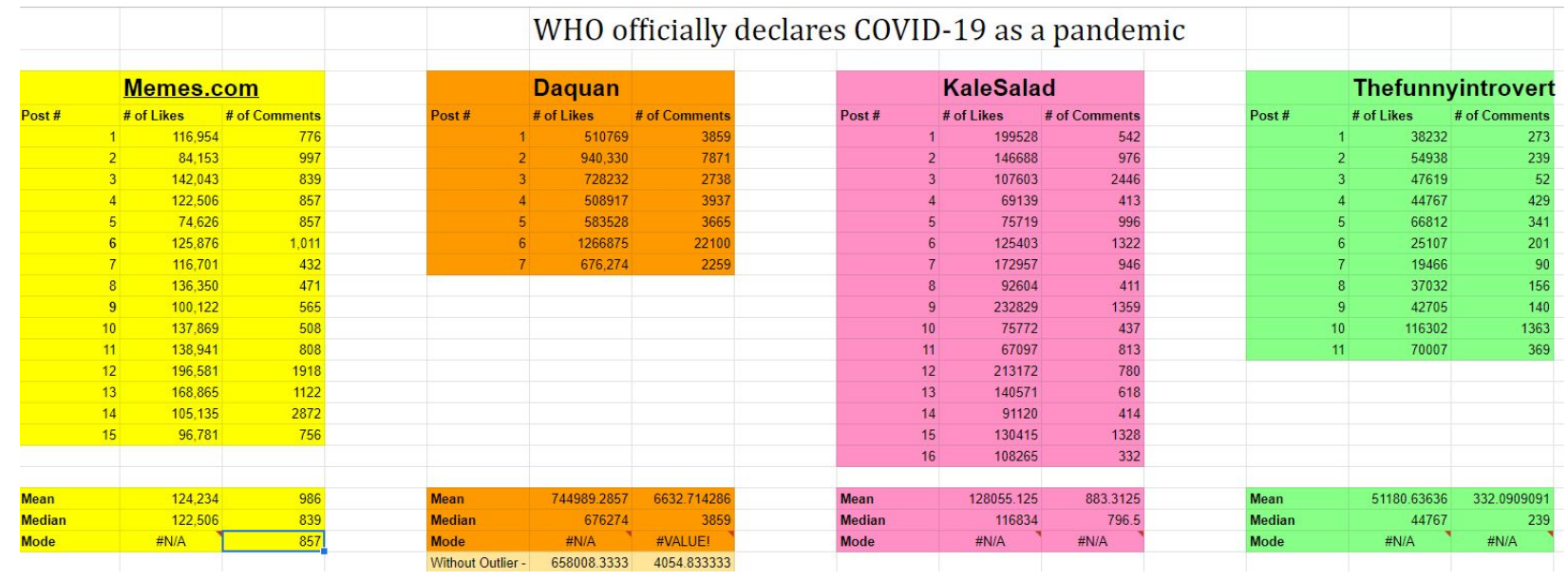

(Figure 1)

The full raw data for this study can be viewed here.

(If the link above does not open, copy and paste the following link for access:

https://docs.google.com/spreadsheets/d/1G0idNuMnOuFMj-lgmRkcn7-gBHx499_CbWWNRGI0HBk/ed it?usp=sharing)

Additionally, memes relevant to the news story and other aspects of COVID-19 were coded and recorded separately with the corresponding engagement metrics. This process was indicated in reference to Figure 2 of this paper (and below for reference) 


\begin{tabular}{|c|c|c|c|c|c|c|}
\hline $\begin{array}{l}\text { Account } \\
\text { Name }\end{array}$ & News Topic & $\begin{array}{c}\text { \# of } \\
\text { Correlating } \\
\text { Posts } \\
\text { (2 Day Span) }\end{array}$ & $\begin{array}{c}\text { \# of } \\
\text { CoVID-19 } \\
\text { Memes }\end{array}$ & \# of Likes & $\begin{array}{c}\text { \# of } \\
\text { Comments }\end{array}$ & $\begin{array}{c}\text { Average } \\
\text { Metrics } \\
\text { (2 Day Span) }\end{array}$ \\
\hline $\begin{array}{l}\text { thefunnyi } \\
\text { ntrovert }\end{array}$ & $\begin{array}{l}\text { Date: March 11, } \\
2020 \\
\text { Story: WHO officially } \\
\text { declares COVID-19 as } \\
\text { a pandemic }\end{array}$ & $\begin{array}{c}2 \\
\text { Voodoo } \\
\text { Diary }\end{array}$ & $\begin{array}{c}3 \\
\text { Tom Hanks } \\
\text { Turkey } \\
\text { Boomer } \\
\text { Remover }\end{array}$ & $\begin{array}{c}42,705 \\
70,007 \\
\\
66,812 \\
37,032 \\
116,302\end{array}$ & $\begin{array}{c}140 \\
369 \\
341 \\
156 \\
1,363\end{array}$ & $\begin{array}{c}\text { Posts: } 11 \\
\text { Average Likes: } \\
51,181 \\
\text { Average } \\
\text { Comments: } 332\end{array}$ \\
\hline $\begin{array}{c}\text { memes.co } \\
\text { m }\end{array}$ & $\begin{array}{l}\text { Date: March 11, } \\
2020 \\
\text { Story: WHO officially } \\
\text { declares COVID-19 as } \\
\text { a pandemic }\end{array}$ & $\begin{array}{l}2 \\
\text { The Simpsons } \\
\text { "Brush yo } \\
\text { Teeth" }\end{array}$ & $\begin{array}{c}3 \\
\text { Flights so } \\
\text { Cheap } \\
\text { Cheap } \\
\text { Flights }\end{array}$ & $\begin{array}{c}196,581 \\
96,781 \\
125,876 \\
100,147\end{array}$ & $\begin{array}{c}1,918 \\
756 \\
1,011 \\
565\end{array}$ & $\begin{array}{c}\text { Posts: } 15 \\
\text { Average Likes: } \\
124,234 \\
\text { Average } \\
\text { Comments: } 986\end{array}$ \\
\hline Daquan & $\begin{array}{l}\text { Date: March 11, } \\
2020\end{array}$ & 2 & 4 & & & $\begin{array}{c}\text { Posts: } 7 \\
\text { Average Likes: }\end{array}$ \\
\hline
\end{tabular}

(Figure 2)

The full raw data for this study can be viewed here.

(If the link above does not open, copy and paste the following link for access:

https://docs.google.com/document/d/1f1UnkKMtQdQB_jyU1zh5X8_zxPYG4Q0zKD0Fsrximps/edit?usp =sharing)

\section{QUALITATIVE ANALYSIS}

For the qualitative analysis conducted for the sample of 29 memes found on Instagram, a content analysis was performed. In order to conduct a thorough content analysis, each meme within the sample was coded based on themes. Figure 3 of this paper (and below for reference) displays how the content analysis was conducted. 


\begin{tabular}{|c|c|c|c|c|c|c|c|c|c|}
\hline \multirow{2}{*}{$\begin{array}{l}\text { Meme } \\
\text { Simpsons Prediction }\end{array}$} & \multicolumn{2}{|c|}{ Theme 1: I Theme 2: } & \multicolumn{3}{|c|}{ Theme 3: ' Theme 4: 1 Theme 5: } & \multicolumn{2}{|c|}{ Theme 6:( Theme 7: } & \multirow[t]{2}{*}{ Theme 8: } & \multirow[t]{2}{*}{ Theme 9: } \\
\hline & 1 & 1 & 1 & & & & & & \\
\hline Boomer Remover & 1 & 1 & 1 & 1 & & 1 & & & \\
\hline WHO let the dogs out & 1 & & 1 & 1 & & & & & \\
\hline Favourite Person & & & & & & & 1 & & 1 \\
\hline Titanic & 1 & & 1 & 1 & & 1 & & & 1 \\
\hline Fight Over Beans & & & 1 & 1 & & & 1 & 1 & 1 \\
\hline Kanye Face & 1 & & 1 & 1 & & & & & 1 \\
\hline ESPN Chess & & & 1 & 1 & & & & 1 & \\
\hline Buy an Island & & & 1 & 1 & & & & & \\
\hline Professor Fail & & & & 1 & & 1 & 1 & & 1 \\
\hline Curd Nerds & & & & 1 & 1 & & & & 1 \\
\hline Hugged Tighter & & & & & & & & & 1 \\
\hline Gag Gift & & & & 1 & & & & & 1 \\
\hline Boyfriend's Job & & & 1 & & & & 1 & 1 & \\
\hline Uno Government & & 1 & 1 & 1 & & 1 & 1 & 1 & \\
\hline Taylor Swift & 1 & & & 1 & & & & & 1 \\
\hline Can't Spend the Night & & & & & 1 & & & & 1 \\
\hline 2020 Planner & & & & 1 & & & & & 1 \\
\hline
\end{tabular}

(Figure 3)

The full raw data for this study can be viewed here.

(If the link above does not open, copy and paste the following link for access:

https://docs.google.com/spreadsheets/d/185DHZYrB-2ZrdyggvKAZXEXQKidb_rc7GEiXupwUK7k/edit ?usp=sharing)

A similar content analysis was conducted for a sample of memes found on the platform Reddit. In order to conduct a thorough content analysis, each meme within the sample was coded based on themes. Figure 4 of this paper (and below for reference) displays how the content analysis was conducted.

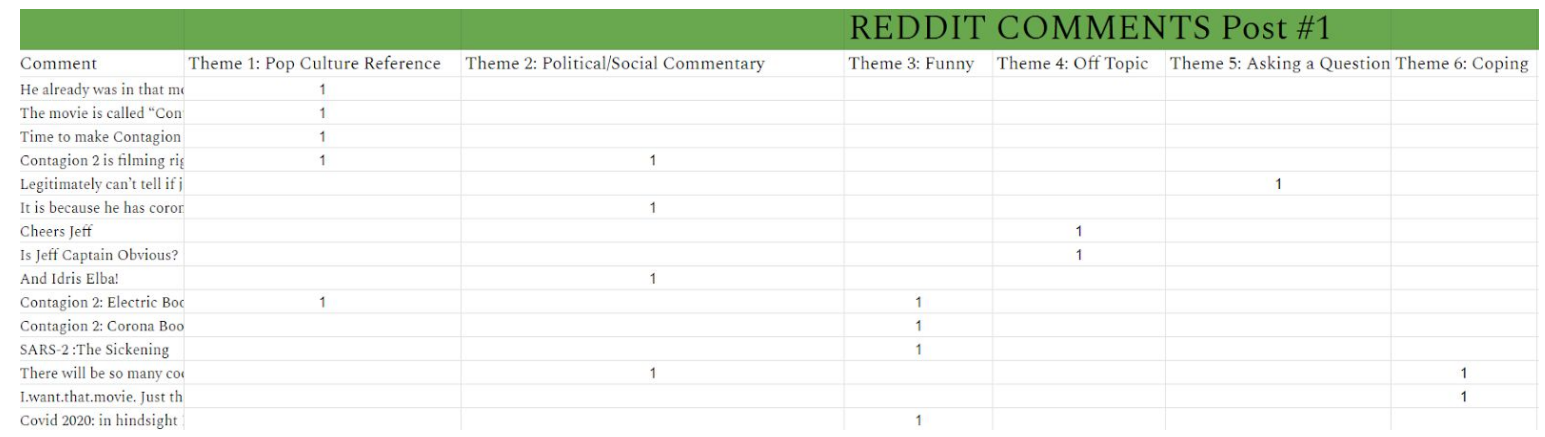

(Figure 4)

The full raw data for this study can be viewed here.

(If the link above does not open, copy and paste the following link for access:

https://docs.google.com/spreadsheets/d/185DHZYrB-2ZrdyggvKAZxEXQKidb_rc7GEiXupwUK7k/edit ?usp=sharing)

\section{APPENDIX C}




\section{All memes mentioned and illustrated within this research paper are referenced below:}

[Danifellan]. (2020, March). Matt Damon Movie (Figure 22) [Online forum post]. Retrieved from https://www.reddit.com/r/CoronavirusMemes/comments/fkozr1/me_irl/

KaleSalad [@kalesalad]. (2020, March 13). [Figure 11]. Retrieved from //https://www.instagram.com/p/B9sAOuvpvrV/

KaleSalad [@kalesalad]. (2020, March 25). [Figure 12]. Retrieved from //https://www.instagram.com/p/B-LBmlyJfOx/

Memes.com [@memes]. (2020, March 16). [Figure 17]. Retrieved from //https://www.instagram.com/p/B9ySBMRllQx/

Memes.com [@memes]. (2020, March 31). [Figure 21]. Retrieved from //https://www.instagram.com/p/B-ZDS5K1HSx/

The Funny Introvert [@thefunnyintrovert]. (2020, March 14). [Figure 10]. Retrieved from //https://www.instagram.com/p/B9uM_KVBO0c/

The Funny Introvert [@thefunnyintrovert]. (2020, March 16). [Figure 18]. Retrieved from //https://www.instagram.com/p/B9zpnophuDe/

The Funny Introvert [@thefunnyintrovert]. (2020, March 14). [Figure 19]. Retrieved from //https://www.instagram.com/p/B9u-lgYBbXv/

The Funny Introvert [@thefunnyintrovert]. (2020, March 12). [Figure 20]. Retrieved from //https://www.instagram.com/p/B9p4b4_h-gS/

\section{Data collection and analysis conducted for the observed 307 memes can be found throughout the following accounts:}

Daquan [@Daquan]. (n.d.). Posts [Instagram profile]. Retrieved August 3, 2020, from https://www.instagram.com/daquan/.

KaleSalad [@kalesalad]. (n.d.). Posts [Instagram profile]. Retrieved August 3, 2020, from https://www.instagram.com/kalesalad/.

Memes.com [@memes]. (n.d.). Posts [Instagram profile]. Retrieved August 3, 2020, from https://www.instagram.com/memes/.

The Funny Introvert [@thefunnyintrovert]. (n.d.). Posts [Instagram profile]. Retrieved August 3, 2020, from https://www.instagram.com/thefunnyintrovert/.

\section{WORKS CITED}


Anderson, M., \& Jiang, J. (2020, May 30). Teens, Social Media \& Technology 2018. Retrieved from https://www.pewresearch.org

Blair, E. (2015). A reflexive exploration of two qualitative data coding techniques. Journal of Methods and Measurement in the Social Sciences, 6(1), 14-29.

doi:https://doi.org/10.2458/v6i1.18772

Collins, B. \& Zadrozny B. Twitter bans 7,000 QAnon accounts, limits 150,000 others as part of broad crackdown. (2020, July 21). NBC News. Retrieved from

https://www.nbcnews.com/tech/tech-news/twitter-bans-7-000-qanon-accounts-limits-150-000-ot hers-n1234541

Fairclough, N. (2013). Critical discourse analysis and critical policy studies. Critical Policy Studies, 7(2), 177-197. https://doi.org/10.1080/19460171.2013.798239

Grundlingh, L. (2018). Memes as speech acts. Social Semiotics, 28(2), 147-168.

Horváth, D., \& Mitev, A. (2016). Internet Memes: The Meteorites of the Online World. Spontaneous Online Content with Corporate Relevance'. Advertising in New Formats and Media. Emerald Group Publishing Limited, 243-262.

Instagram by the Numbers: Stats, Demographics \& Fun Facts. (2020, February 10). Retrieved May 15, 2020, from https:/www.omnicoreagency.com/instagram-statistics/

Kien, G. (2013). Media memes and prosumerist ethics: Notes toward a theoretical examination of memetic audience behavior. Cultural Critical Methodologies, 13(6), 554-561.

Kien, G. (2019). Communicating with memes : Consequences in post-truth civilization. Retrieved from https://ebookcentral-proquest-com.ezproxy.lib.ryerson.ca

Literat, I., \& van den Berg, S. (2019). Buy memes low, sell memes high: Vernacular criticism and collective negotiations of value on Reddit's MemeEconomy. Information, Communication \& Society, 22(2), 232-249.

Marcus, O. R., \& Singer, M. (2017). Loving Ebola-chan: Internet memes in an epidemic. Media, Culture \& Society, 39(3), 341-356.

Malina, M. A., Hanne S.O. Nørreklit, \& Selto, F. H. (2011). Lessons learned: Advantages and disadvantages of mixed method research. Qualitative Research in Accounting and Management, 8(1), 59-71. doi:http://dx.doi.org.ezproxy.lib.ryerson.ca/10.1108/11766091111124702

Merriam-Webster. (n.d.). Meme. In Merriam-Webster.com dictionary. Retrieved July 30, 2020, from https://www.merriam-webster.com/dictionary/meme

The Demographics of Reddit: Who Uses the Site? (2020, April 17). Retrieved from 
https://social.techjunkie.com/demographics-reddit/

Van Dijk, T. A. (1993). Principles of Critical Discourse Analysis. Discourse \& Society, 4(2), 249-283. https://doi.org/10.1177/0957926593004002006

Weng, L., Flammini, A., Vespignani, A., \& Menczer, F. (2012). Competition among memes in a world with limited attention. Scientific reports, 2, 335.

Wiggins, B. E., \& Bowers, G. B. (2015). Memes as genre: A structurational analysis of the memescape. new media \& society, 17(11), 1886-1906.

Wu, J. (2011). Understanding Interdiscursivity: A Pragmatic Model?.

Yang, F. (2016). Rethinking China's Internet censorship: The practice of recoding and the politics of visibility. New Media \& Society, 18(7), 1364-1381. 